\title{
Integrative taxonomy unravels cryptic diversity in the Paratrichodorus hispanus-group complex and resolves two new species of the genus and the molecular phylogeny of the family (Nematoda: Trichodoridae)
}

\author{
WILFRIDA DECRAEMER ${ }^{1}$, CAROLINA CANTALAPIEDRA-NAVARRETE ${ }^{2}$, \\ ANTONIO ARCHIDONA-YUSTE ${ }^{2}$, INGRID VARELA-BENAVIDES ${ }^{3}$, \\ CARLOS GUTIÉRREZ-GUTIÉRREZ ${ }^{4,5}$, PABLO CASTILLO $^{2, \bullet}$ AND \\ JUAN E. PALOMARES-RIUS ${ }^{2 *, \oplus}$
}

${ }^{1}$ Ghent University, Department of Biology, Ledeganckstraat 35, B-9000 Ghent, Belgium

${ }^{2}$ Institute for Sustainable Agriculture (IAS), Spanish National Research Council (CSIC), Avda.

Menendez Pidal s/n, 14004 Córdoba, Spain

${ }^{3}$ Laboratorio de Nematología, Instituto Tecnológico de Costa Rica sede San Carlos, Apartado postal: $223-$ 21001, Alajuela, San Carlos, Costa Rica

${ }^{4}$ NemaLab / ICAAM, Instituto de Ciências Agrárias e Ambientais Mediterrânicas \& Dept. de 16 Biologia, Universidade de Évora, Núcleo da Mitra, Ap. 94, 7002-554 Évora, Portugal

${ }_{5}^{5}$ Dept. Protección Vegetal, Estación Experimental de Santa Catalina, Instituto Nacional Autonómico de Investigaciones Agropecuarias (INIAP), Panamericana Sur s/n, Ap. 1701-340, Machachi, Ecuador /

Project Prometeo - SENESCYT, Quito 170184, Ecuador

Received 12 January 2018; revised 29 June 2018; accepted for publication 10 August 2018

The genus Paratrichodorus currently comprises 26 species of polyphagous ectoparasitic plant nematodes that are distributed worldwide and include a few tobravirus vector species. Paratrichodorus is one of the most difficult genera for species identification because it is phenotypically conserved, with morphometric characters that often overlap between species. From 2003 to 2016, nematode surveys were conducted in southern Spain, and the present study addresses 22 nematode populations of the Paratrichodorus hispanus group. Among them, two new species, Paratrichodorus almadenensis sp. nov. and Paratrichodorus ramblensis sp. nov., are described here morphologically and molecularly and compared with topotype specimens of Paratrichodorus hispanus. Furthermore, this analysis was complemented with the first molecular data for this species complex and for the genus Monotrichodorus. The phylogeny of family Trichodoridae showed the relationship of Monotrichodorus with Trichodorus, both of which are phylogenetically associated with the $P$. hispanus species group, including the two newly described species. Several phylogenetic hypotheses about the monophyly of the genera with molecular data composing the family Trichodoridae were tested using Shimodaira-Hasegawa and approximately unbiased tests. This work also includes the first observations of copulatory plugs in Paratrichodorus, which clearly differ from the different types of mating plugs described in Trichodorus species.

ADDITIONAL KEYWORDS: approximately unbiased and Shimodaira-Hasegawa tests - Bayesian inference cryptic species - cytochrome oxidase $c$ subunit 1 - expansion domains of the large ribosomal subunit (28S) internal transcriber spacer, rRNA - stubby root nematodes.

\footnotetext{
*Corresponding author. E-mail: palomaresje@ias.csic.es [Version of Record, published online 22 October 2018; http://zoobank.org/urn:lsid:zoobank.org:pub:48B9F19AF2F5-4044-86C1-11A9B78E4503]
}

\section{INTRODUCTION}

Stubby root nematodes of the family Trichodoridae include 111 species grouped within six genera, i.e. three didelphic genera and three monodelphic 
genera (Decraemer, 1995; Decraemer \& Robbins, 2007). Paratrichodorus is the second most speciesrich didelphic genus (26 species) after Trichodorus (65 species), but seven of the species in the former have been transferred to the genus Nanidorus upon the recognition of the latter as a valid genus (Duarte et al., 2010). The other monodelphic-prodelphic genera are Monotrichodorus (five species), Allotrichodorus (six species) and Ecuadorus (two species), which are restricted to an area in Central America and the northern part of South America (Decraemer \& Robbins, 2007).

Within the family Trichodoridae, identification to the species level is hampered by the largely conserved morphology, overlapping morphometrics and co-occurrence of more than one trichodorid species in the same soil sample. Another issue is the difficulty in properly fixing trichodorid nematodes, particularly Paratrichodorus species, and the degradation of type specimens in nematode collections that result in outer and/or inner disturbance of structures and systems, particularly to the pharynx, including the stylet (onchium and onchiophore) and the spicules. One more restriction includes the need for specimens to be in a lateral position for correct observation of the main diagnostic features, such as the shape of the vagina and sclerotized pieces in the female and spicule shape in the male.

The present contribution focuses on the genus Paratrichodorus in the framework of continuous surveys for Trichodoridae in cultivated and natural environments in southern Spain since 2003. A previous paper on the genus Trichodorus, including seven new species, was published by Decraemer et al. (2013). The main diagnostic morphological characters for Paratrichodorus at the species level are as follows: (1) in males, the shape, ornamentation and length of the spicules, the number and position of precloacal supplements within the region of retracted spicules, the number and position of ventromedian cervical papillae and, to a lesser extent, tail shape and length, the position of the postcloacal papillae and the size of the bursa; and (2) in females, the size, shape, position and orientation of the vaginal sclerotized pieces (pars refringens vaginae) in the optical section of the sclerotized ring and, to a lesser extent, the size and shape of the vagina and the presence of sublateral body pores. Given that the information provided by morphological features may be rather limited, morphometric data, such as body length and the lengths of the onchiostyle and spicule, are more important as diagnostic features than in Trichodorus (Decraemer, 1995). However, morphometric data within Paratrichodorus should be considered with caution because fixation results in contraction of the outer body cuticle and/or the inner organs (Sturhan, 1985).

López Pérez et al. (2001) revised the Trichodoridae in Spain and found 18 species in the Iberian Peninsula and Islands, including six Paratrichodorus species: $P$. allius (Jensen, 1963) Siddiqi, 1974; $P$. anemones (Loof, 1965) Siddiqi, 1974; P. hispanus Roca \& Arias, 1986; P. pachydermus (Seinhorst, 1954) Siddiqi, 1974; P. porosus (Allen, 1957) Siddiqi, 1974; and P. teres (Hooper, 1962) Siddiqi, 1974; as well as three Nanidorus species: N. acutus (Bird, 1967) Siddiqi, 1974; N. minor (Colbran, 1956) Siddiqi, 1974; and $N$. nanus (Allen, 1957) Siddiqi, 1974. Paratrichodorus teres and N. minor were recorded only from the Canary Islands, and a previous report of Paratrichodorus cf. acutus could not be confirmed. Paratrichodorus hispanus appeared widespread in Spain and northern Portugal, whereas the other species were strongly localized (viz. P. allius and P. anemones), and $P$. porosus was found only in the Azores and Madeira Archipelagos. Duarte et al. (2010) reported 16 trichodorid species from Portugal, including Paratrichodorus divergens Almeida, Santos, Abrantes \& Decraemer, 2005 as the most recently described species of this genus in Portugal. The authors also recorded the presence of $P$. porosus in continental Europe for the first time. Paratrichodorus teres has been recorded in mainland Portugal. Paratrichodorus anemones and P. pachydermus are characteristic of temperate environments; they are widespread in the UK and in western and northern Europe. At present, seven out of 26 Paratrichodorus species $(27 \%)$ have been recorded in the Iberian Peninsula. The major problem posed by nematodes of Trichodoridae to crops is that they are vectors of tobraviruses. Currently, nine Paratrichodorus species (26\%) are known to be natural tobravirus vectors (Decraemer \& Robbins, 2007). Except for P. porosus, all Paratrichodorus species known at present from the Iberian Peninsula are natural vectors of these plant viruses.

Molecular characterization based on the 18S rRNA gene by Kumari \& Subbotin (2012) showed two main clades of Paratrichodorus species: (1) clade 1 with two subclades, one with species with undulating spicular calomus (all present in the Iberian Peninsula, $P$. anemones, $P$. hispanus complex, Paratrichodorus sp. B, Paratrichodorus sp. C and $P$. divergens) and one with two species frequent in temperate latitudes with non-undulating spicules $(P$. pachydermus and $P$. macrostylus); and (2) clade 2 with the species $P$. allius, $P$. porosus and $P$. teres, for which males can be rare; the first two species are more common in warmer climates. This observation confirms the subdivision found by Duarte et al. (2010) based on the 18S rRNA gene.

Hitherto, no studies with molecular data illustrate the phylogenetic relationship between the monodelphic and didelphic genera in the family Trichodoridae that share important morphological features. For example, the variable swelling of the body cuticle and an approximately straight body appearance in addition to the presence of a bursa and copulatory muscles restricted to the spicule region in the male are shared between 
Paratrichodorus and Allotrichodorus, or body cuticle less or not swollen, male appearance more or less J-shaped, i.e. with curved posterior body region, and bursa absent (except in Trichodorus cylindricus and Trichodorus paracedarus) and copulatory muscles extending shortly to far beyond the region of retracted spicules are shared between Trichodorus and Monotrichodorus (Decraemer \& De Almeida, 1997). The didelphic genus Ecuadorus is known only from parthenogenetic females and seems to occupy a somewhat intermediate position between the didelphic genera, more specifically Nanidorus (short vagina, small/minute vaginal sclerotized pieces, a transverse slit-like vulva, no spermatheca and no caudal pores) and the monodelphic genera [single ovary and uterus with a transverse slit-like vulva (Allotrichodorus and Monotrichodorus) but with a longer vagina, presence of spermatheca and caudal pores (Allotrichodorus and Monotrichodorus) and a Neotropical distribution] (Siddiqi, 2002; Decraemer \& Robbins, 2007). Unfortunately, only hypotheses and no data are available to clarify the evolution of monodelphic species. According to Siddiqi (2002), Nanidorus, with a worldwide distribution, could have originated in the Neotropical region, but Decraemer \& Robbins (2007) proposed that monodelphic genera developed from an ancestral stock immediately before or immediately after the separation of current South America during the Cretaceous period based on a transformation series: $N$. minor (didelphic, vulva at 50-64\%), N. caribensis (didelphic, vulva at 60-64\%) and Ecuadorus spp. (monodelphic, vulva at $60-68 \%$ ).

The objectives of the present work are as follows: (1) to study, with a combined morphological and molecular approach, Paratrichodorus spp. populations in nematode surveys from 2003 to 2016 from the Paratrichodorus hispanus group using paratype and topotype specimens and the description of two new species for the genus; and (2) to provide, for the first time, molecular data and morphological characterization of Monotrichodorus species from Costa Rica and Ecuador and their phylogenetic relationship with the other genera of the family Trichodoridae with molecular data available.

\section{MATERIAL AND METHODS}

\section{NEMATODE POPULATIONS AND MORPHOLOGICAL STUDIES}

From 2003 to 2016, nematode surveys were conducted during the spring season in cultivated olive (Olea europaea subsp. europaea) and wild olive (Olea europaea subsp. silvestris) in southern Spain, and incidental samples were collected from other host plants (Table 1). Additionally, samples were collected from Costa Rica and Ecuador (Table 1) by two of the authors
(I.V.-B. and C.G.-G., respectively), including four monodelphic populations from forest naranjilla (Solanum quitoense Lamarck) and potato (Solanum tuberosum L.). However, given that only one adult female specimen was detected for each Ecuadorian population, those samples were combined with some juveniles for DNA extraction and sequencing; the species could not be identified to species level, but clearly belong to the genus Monotrichodorus. Samples were collected with a hoe from the upper $50 \mathrm{~cm}$ of soil under four or five plants that were arbitrarily chosen in each locality, and nematodes were extracted from $500 \mathrm{~cm}^{3}$ of soil by centrifugal flotation (Coolen, 1979). In some cases, additional soil samples were collected afterwards from the same locality to obtain the number of specimens necessary for morphological and/or molecular identification.

Specimens used for morphological studies were killed by gentle heat, fixed in a solution of $4 \%$ formaldehyde plus $1 \%$ propionic acid and processed to pure glycerine using the method of Seinhorst (1966). Specimens were examined using a Zeiss III compound microscope [Consejo Superior de Investigaciones Científicas (CSIC)-IAS, Spain], and drawings were made and photomicrographs taken using a Nikon Echpx microscope, with Nomarski differential interference contrast at powers up to $\times 1000$ magnification, connected to a Nikon camera and DS-L4 tablet (Ghent University, Ghent, Belgium). Measurements were made using a drawing tube attached to a light microscope, unless otherwise indicated in the text. All abbreviations are as defined by Siddiqi (1973). In addition, a comparative morphological study was conducted of $P$. hispanus type specimens kindly provided by Dr A. Navas from the Nematode Collection of the Museo Nacional de Ciencias Naturales, CSIC, Madrid, Spain.

\section{EXTRACTION OF DNA, PCR AND SEQUENCING}

To avoid mistakes in case of mixed populations in the same sample, two live nematodes from each sample were temporarily mounted in a drop of $1 \mathrm{M} \mathrm{NaCl}$ containing glass beads, and diagnostic measurements and photomicrographs were taken to confirm the identity. However, these measurements were not used for morphometrical studies or analyses. The slides were then dismantled, and the DNA was extracted. Nematode DNA was extracted from single individuals, and some PCR assays were conducted as described by Subbotin et al. (2000). A single nematode specimen was transferred to an Eppendorf tube containing $16 \mu \mathrm{L}$ dd $_{2} \mathrm{O}, 2 \mu \mathrm{L} 10 \times$ PCR buffer and $2 \mu \mathrm{L}$ proteinase K $(600 \mu \mathrm{g} / \mathrm{mL})$ (Promega, Benelux, The Netherlands) and crushed with a Vibro Mixer microhomogenizer (Zürich, Switzerland) for $2 \mathrm{~min}$. The tubes were incubated at $65{ }^{\circ} \mathrm{C}(1 \mathrm{~h})$, then at $95{ }^{\circ} \mathrm{C}$ 


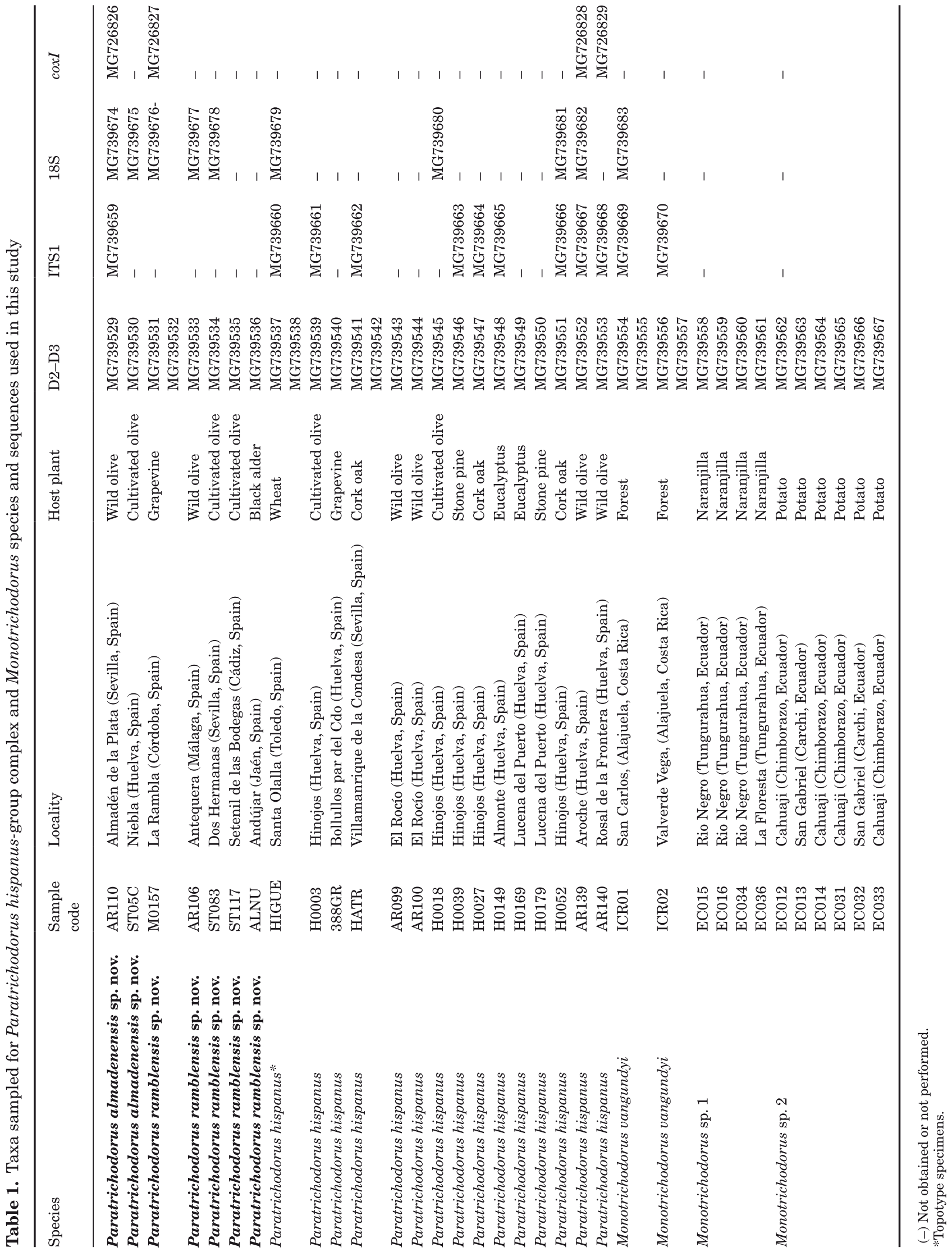


(15 min), and finally at $-80^{\circ} \mathrm{C}(15 \mathrm{~min})$. One microlitre of extracted DNA was transferred to an Eppendorf tube containing $2.5 \mu \mathrm{L} 10 \times \mathrm{NH}_{4}$ reaction buffer, $0.75 \mu \mathrm{L}$ $\mathrm{MgCl}_{2}(50 \mathrm{mM}), 0.25 \mu \mathrm{L}$ dNTPs mixture (10 mM each), $0.75 \mu \mathrm{L}$ of each primer $(10 \mathrm{mM}), 0.2 \mu \mathrm{L}$ BIOTAQ DNA Polymerase (Bioline, UK) and $\mathrm{dd}_{2} \mathrm{O}$ to a final volume of $25 \mu \mathrm{L}$. The D2-D3 expansion domains of the 28S rRNA gene were amplified using the primers D2A (5'-ACAAGTACCGTGAGGGAAAGTTG-3') and D3B (5'-TCGGAAGGAACCAGCTACTA-3') (Nunn, 1992). The ITS1 region was amplified using forward primer BL18 (5'-CCCGTCGMTACTACCGATT-3') and reverse primer 5818 (5'-ACGARCCGAGTGATCCAC-3') (Boutsikaetal.,2004a), and the partial18SrRNAgenewas amplified as described by Holterman et al. (2006) using primers $988 \mathrm{~F}$ (5'-CTCAAAGATTAAGCCATGC-3'), $1912 \mathrm{R}$ (5'-TTTACGGTCAGAACTAGGG-3'), $1813 \mathrm{~F}\left(5^{\prime}-\mathrm{CTGCGTGAGAGGTGAAAT}^{\prime} 3^{\prime}\right)$ and $2646 \mathrm{R}$ (5'-GCTACCTTGTTACGACTTTT-3') (Holterman et al., 2006). Finally, primers COI-F5 (5'-AATWTWGGTGTTGGAACTTCTTGAAC-3') and COIR5 (5'- CTTAAAACATAATGRAAATGW GCWACWACATAATAAGTATC-3') (Powers et al., 2014) were used to amplify the portion of coxI in P. hispanus populations, and COIF (5'-GATTTTTTGGKCATCCWGARG-3') (He et al., 2005) and COIR5 were used for the other species.

The PCR cycle conditions for ribosomal DNA (rDNA) markers were as follows: one cycle of $94{ }^{\circ} \mathrm{C}$ for 2 min, followed by 35 cycles of $94^{\circ} \mathrm{C}$ for $30 \mathrm{~s}$; annealing temperatures of 55,50 and $54{ }^{\circ} \mathrm{C}$ for $45 \mathrm{~s}$ for the D2-D3 expansion domains of the 28S rRNA, ITS1 and partial 18S rRNA genes, respectively; $72^{\circ} \mathrm{C}$ for $3 \mathrm{~min}$ and, finally, one cycle of $72^{\circ} \mathrm{C}$ for $5 \mathrm{~min}$. The PCR conditions for mitochondrial DNA consisted of denaturation at $94{ }^{\circ} \mathrm{C}$ for 5 min followed by 40 cycles of denaturation at $94{ }^{\circ} \mathrm{C}$ for $30 \mathrm{~s}$, annealing at $51{ }^{\circ} \mathrm{C}$ for $30 \mathrm{~s}$, extension at $68^{\circ} \mathrm{C}$ for $1.5 \mathrm{~min}$ and a final extension at $68^{\circ} \mathrm{C}$ for $5 \mathrm{~min}$. The PCR products were purified after amplification using ExoSAP-IT (Affmetrix, USB products) and used for direct sequencing in both directions using the above primers. The resulting products were purified and run on a DNA multicapillary sequencer (model 3130XL genetic analyser; Applied Biosystems, Foster City, CA, USA), using the BigDye Terminator Sequencing Kit v.3.1 (Applied Biosystems), at the Stab Vida sequencing facilities (Caparica, Portugal). The newly obtained sequences were submitted to the GenBank database under the accession numbers indicated on the phylogenetic trees and in Table 1.

\section{PHYLOGENETIC ANALYSIS}

The D2-D3 expansion domains of the 28S rRNA gene and partial 18S rRNA gene sequences from $P$. hispanus group species and Monotrichodorus spp., in addition to different accessions belonging to the Trichodoridae family that are available in GenBank, were used for phylogenetic reconstruction. Outgroup taxa for each data set were chosen according to previously published data (Kumari \& Subbotin, 2012) that include all the molecular variation in the analysed sequences, and they were found to be outside the family Trichodoridae in wider phylogenies (van Megen et al. 2009). The newly obtained and published sequences for each gene were aligned using MAFFT (Katoh et al., 2002) with default parameters, and the sequence alignments were edited manually using BioEdit (Hall, 1999). The best-fitted model of DNA evolution was obtained using jModelTest v.2.1.7 (Darriba et al., 2012) with the Akaike information criterion (AIC). The base frequency, the proportion of invariable sites, and the $\gamma$-distribution shape parameters and substitution rates in the AIC-supported model were then used in phylogenetic analyses. Phylogenetic analyses were performed with maximum likelihood (ML) using PAUP* 4b10 (Swofford, 2003) and Bayesian inference (BI) using MrBayes v.3.1.2 (Ronquist \& Huelsenbeck, 2003). Bayesian inference analysis under a general time reversible of invariable sites and a $\gamma$-shaped distribution $(\mathrm{GTR}+\mathrm{I}+\mathrm{G})$ model for the D2-D3 expansion domains of the 28S rRNA gene, and a transition of invariable sites and $\gamma$-shaped distribution $(\mathrm{TIM}+\mathrm{IG})$ model for the partial 18S rRNA gene were run with four chains for $1 \times 10^{6}$ generations. The Markov chains were sampled at intervals of 100 generations. Two runs were performed for each analysis, and after discarding burn-in samples and evaluating convergence, the remaining samples were retained for further analyses. The topologies were used to generate a 50\% majority rule consensus tree, and posterior probabilities (PP) and bootstrap support (BS) are given for appropriate clades. Trees were visualized using TreeView (Page, 1996). In the ML analysis, the estimated support for each node was obtained by bootstrap analysis with 100 fast-step replicates. It was not possible to perform phylogenetic analyses from the ITS1 region and partial coxI. The scarce similarity and low coverage detected among the ITS1 region from Paratrichodorus spp. hampered the construction of its phylogeny. In contrast, there was only one Trichodoridae sp. with coxI available in GenBank, Trichodorus obtusus; therefore, the phylogenetic reconstruction could not be carried out for this marker.

For the testing of hypotheses, two additional data sets (the D2-D3 expansion domains of the 28S rRNA gene and partial 18S rRNA gene) were created using selected sequences (at least one sequence from each clade) from four genera with available molecular data (Trichodorus, Nanidorus, Monotrichodorus and Paratrichodorus; Supporting Information, Table S1). 
We used the Shimodaira-Hasegawa $(\mathrm{SH})$ test with ML trees as implemented in PAUP* (Shimodaira \& Hasegawa, 1999; Swofford, 2003) and the RELL option to compare different hypotheses with the unconstrained ML tree, as follows: (1) all Trichodoridae genera separated into different clades; (2) Trichodorus and Nanidorus in one clade and the other separated; and (3) Trichodorus in one clade. The $P$-value of the approximately unbiased (AU) test using the multiscale bootstrap technique was calculated using the program Consel (Shimodaira \& Hasegawa, 2001) for the same trees used in the $\mathrm{SH}$ test.

\section{RESULTS}

\section{SYSTEMATICS}

\section{GENUS PARATRICHODORUS SIDDIQI, 1974}

Amended diagnosis: Cuticle usually clearly swollen when fixed or heat-killed. Female reproductive system didelphic-amphidelphic. Lateral body pores present in $\sim 50 \%$ of the species but rarely located post-advulvar; body pores exceptionally ventral or sub-ventral in position. Vagina short (about onethird of corresponding body diameter), constrictor muscles inconspicuous, vaginal sclerotization small to inconspicuous in lateral view. Males rare or unknown in $\sim 25 \%$ of the species. Ventromedian cervical papillae in male absent or, if present, usually one papilla near the secretory-excretory (S-E) pore, exceptionally with two. Lateral cervical pores absent or, if present, usually one pair near onchiostyle base or S-E pore. Male tail region straight; caudal alae present, obscure to distinct. Sperm cells variable, large size with large sausage-shaped nucleus, medium-sized with rounded nucleus, small with small oval to rounded nucleus or thread-like (degenerated) with nucleus obscure, rarely sperm exceptionally long, fusiform with elongated nucleus. Oblique copulatory muscles poorly developed and restricted to spicule region; capsule of spicule suspensor muscles inconspicuous. Spicules usually straight and blade about equally wide and often finely striated; no bristles nor velum. One to three ventromedian precloacal supplements, exceptionally four; usually two supplements within spicule region, rarely with a single supplement. Usually one pair, rarely two, subventral postcloacal papillae. A pair of caudal pores present, rarely absent. Type species: Paratrichodorus tunisiensis (Siddiqi, 1963) Siddiqi, 1974.

Remarks: Species determination of the P. hispanus group was based upon the integrative approach, applying morphological and molecular methods in order to unravel potential cryptic species diversity. The Spanish samples revealed two main species groups among the Paratrichodorus species found: (1) species possessing large sperm cells with a sausage-shaped or rounded nucleus; and (2) species with small or non-functional sperm cells. Within the first species group, all species have males with undulating spicular calomus; the group is referred to as the $P$. hispanus species group. The second group of species, or the $P$. allius species group, will be dealt in detail in a separate paper.

\section{PARATRICHODORUS HISPANUS SPECIES GROUP}

\section{PARATRICHODORUS HISPANUS ROCA \& ARIAS, 1986}

Topotypes (Figs 1-4; Tables 2-4)

Paratypes and topotypes: Two slides, each with a male and a female paratype, have been kindly put at our disposal by Dr Alfonso Navas, from the Nematode Collection of the Museo Nacional de Ciencias Naturales, Consejo Superior de Investigaciones Científicas, Madrid, Spain. Topotype material was collected by one of the authors (A.A.-Y.), containing four slides with in total 14 males, 18 females and five juveniles, several not suitable for measuring (bad fixation or in oblique or dorsoventral position). Topotype specimens were extracted from soil samples collected from rhizosphere of wheat at Santa Olalla, Toledo province, central Spain $\left(40^{\prime} .05^{\prime} 83.93^{\prime \prime} \mathrm{N}, 4^{\prime} 42^{\prime} 57.44^{\prime \prime} \mathrm{W}\right)$, mounted in pure glycerine and deposited in the Nematode Collection of the Ghent University, Ghent, Belgium (slide numbers HIGUE-01-HIGUE-12).

Description of male: One paratype male and one paratype female in lateral position, one paratype male in ventral view and one paratype female in oblique dorsal view. The body cuticle was partly swollen, but the specimens were still in rather good condition. They agreed well with the original description. However, owing to the swelling of the body cuticle, no lateral body pores could be observed in the female. Additional measurements are provided of the onchium, pharyngostom, the pharynx measured along the lumen, the length of the ovaries and genital branches, the G1 (tail/length of the anterior ovary) and G2 (tail/ length of the posterior ovary) ratios and the length of vaginal sclerotized pieces in optical section $(2.3 \mu \mathrm{m})$. In the paratype male, spicules with narrower undulated calomus $1.5 \mu \mathrm{m}$ wide, maximum width of the blade was $3.0 \mu \mathrm{m}$. In addition to the original description, photomicrographs and drawings are provided of the paratype specimens investigated (Figs 2, 3). 
A
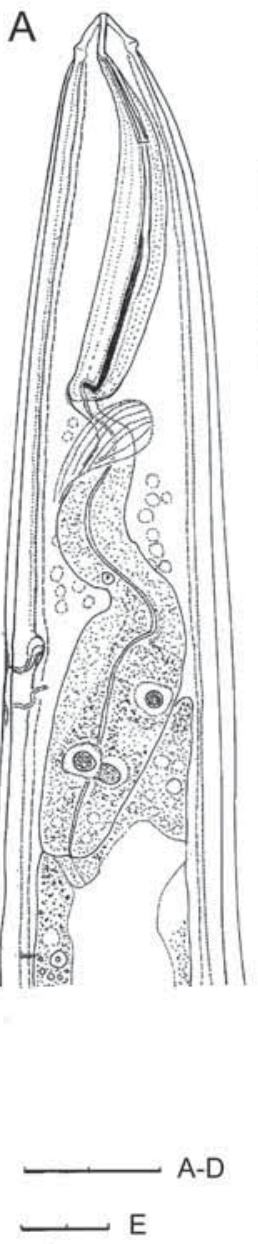

C

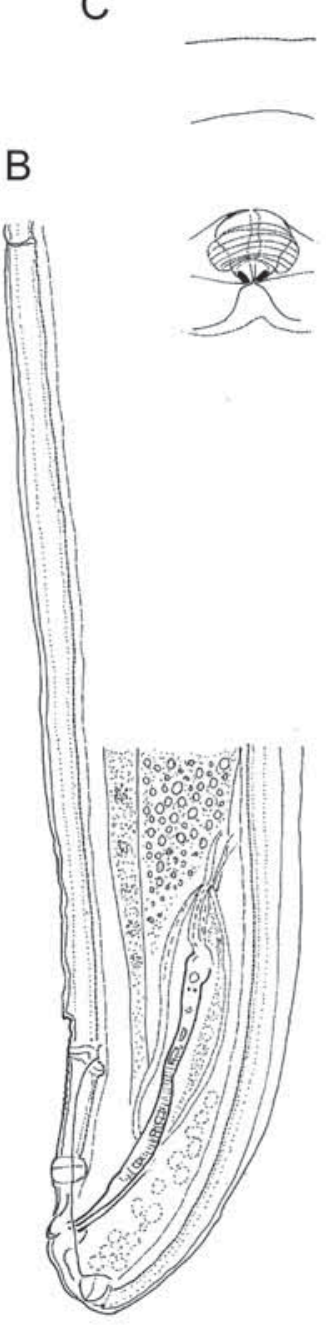

D<smiles>C1CCCCC1</smiles>

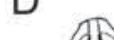
An

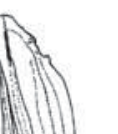

(2)
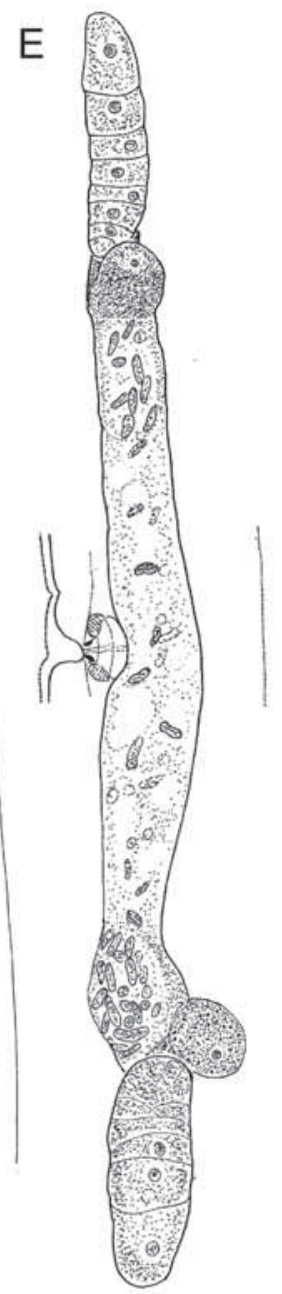

Figure 1. Line drawings of Paratrichodorus hispanus paratypes. A, B, male. A, neck region. B, posterior body region. C-E, female. C, vaginal region. D, neck region. $\mathrm{E}$, reproductive system. Scale bars: $20 \mu \mathrm{m}$ in A-D; $10 \mu \mathrm{m}$ in $\mathrm{E}$.

Description of female: Female largely as in male; S-E pore at level of anterior pharyngeal bulb. Vagina short, rhomboid [average $12.0 \mu \mathrm{m}$ long, i.e. $30 \%$ of cloacal body width (cbw)], usually more or less indented at mid-level owing to contraction of well-developed vaginal constrictor muscles. Vaginal sclerotized pieces, oblique, small droplike to rounded triangular (average $1.7 \mu \mathrm{m}$ ), with distal tips very close (average $1.5 \mu \mathrm{m}$ ). One postvulval lateral body pore observed $36-88 \mu \mathrm{m}(54.0 \mu \mathrm{m}$ on average) from vulva, and in one specimen with uteri filled with sperm, a secretion plug was observed within the vagina. Vulva can be sunk in some specimens, and in ventral view the vulva appears as a transverse slit at the body surface, but a clear pore at the level of the sclerotized ring; slightly more inwards the lumen appears star shaped in transverse optical section (Fig. 4).
Remarks: Male and female topotype specimens are clearly shorter than the paratype material, with body size outside the lower range provided for the type material. This also results in lower values for most other features. The length of the onchiostyle is also shorter, although with overlap at the lower range of the type specimens, similar for the spicule length. All specimens show a dorsal overlap of the pharynx by the intestine, and the nerve ring is adjacent to the base of the pharyngostom. In males, the single ventromedian cervical papilla (CP1) is non-protruding and located shortly (average $6.0 \mu \mathrm{m}$ ) anterior to the S-E pore, located at the level of the posterior isthmus region. Lateral cervical pores (LP) are present at the level of CP1 or immediately anterior to it. Germinal zone of testis varies from short to medium-sized and sperm nucleus 

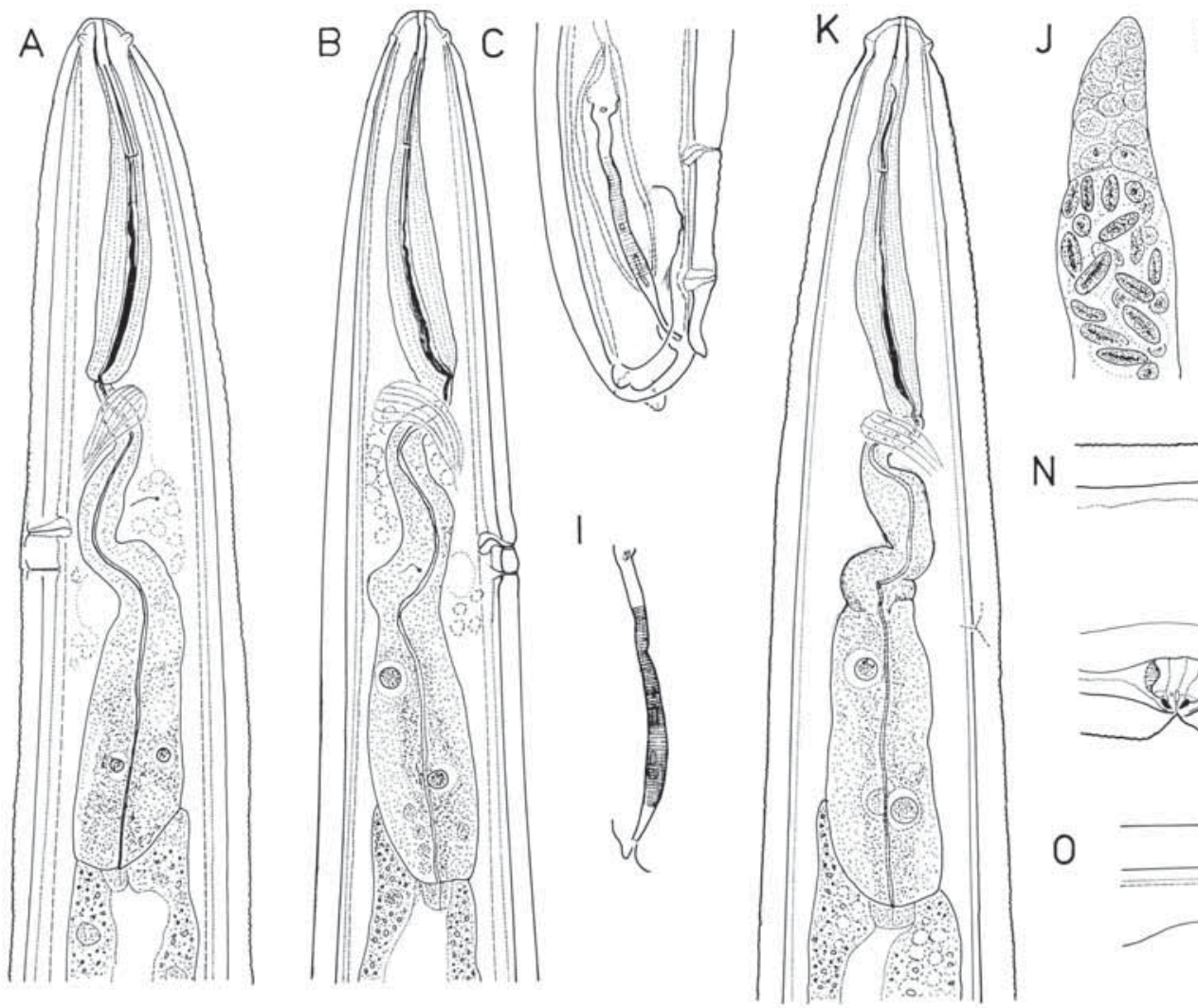

M
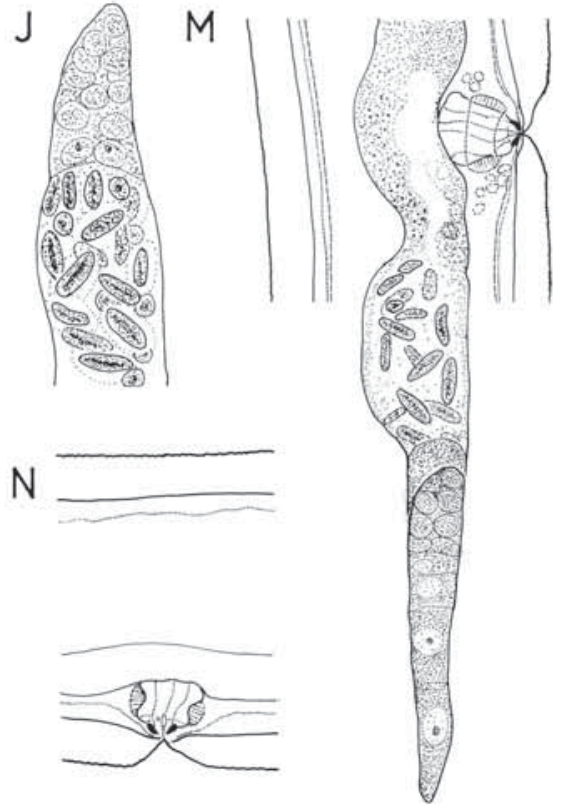

Figure 2. Line drawings of Paratrichodorus hispanus, topotypes. A-J, males. A, B, neck region. C-H, posterior body region with copulatory apparatus. I, spicule. J, testis, anterior part. L-O, females. L, female tail region. M, posterior branch of reproductive system and vagina; $\mathrm{N}-\mathrm{P}$, vaginal region. Scale bar: $10 \mu \mathrm{m}$.

ㄷ 2018 The Linnean Society of London, Zoological Journal of the Linnean Society, 2019, 185, 656-692 

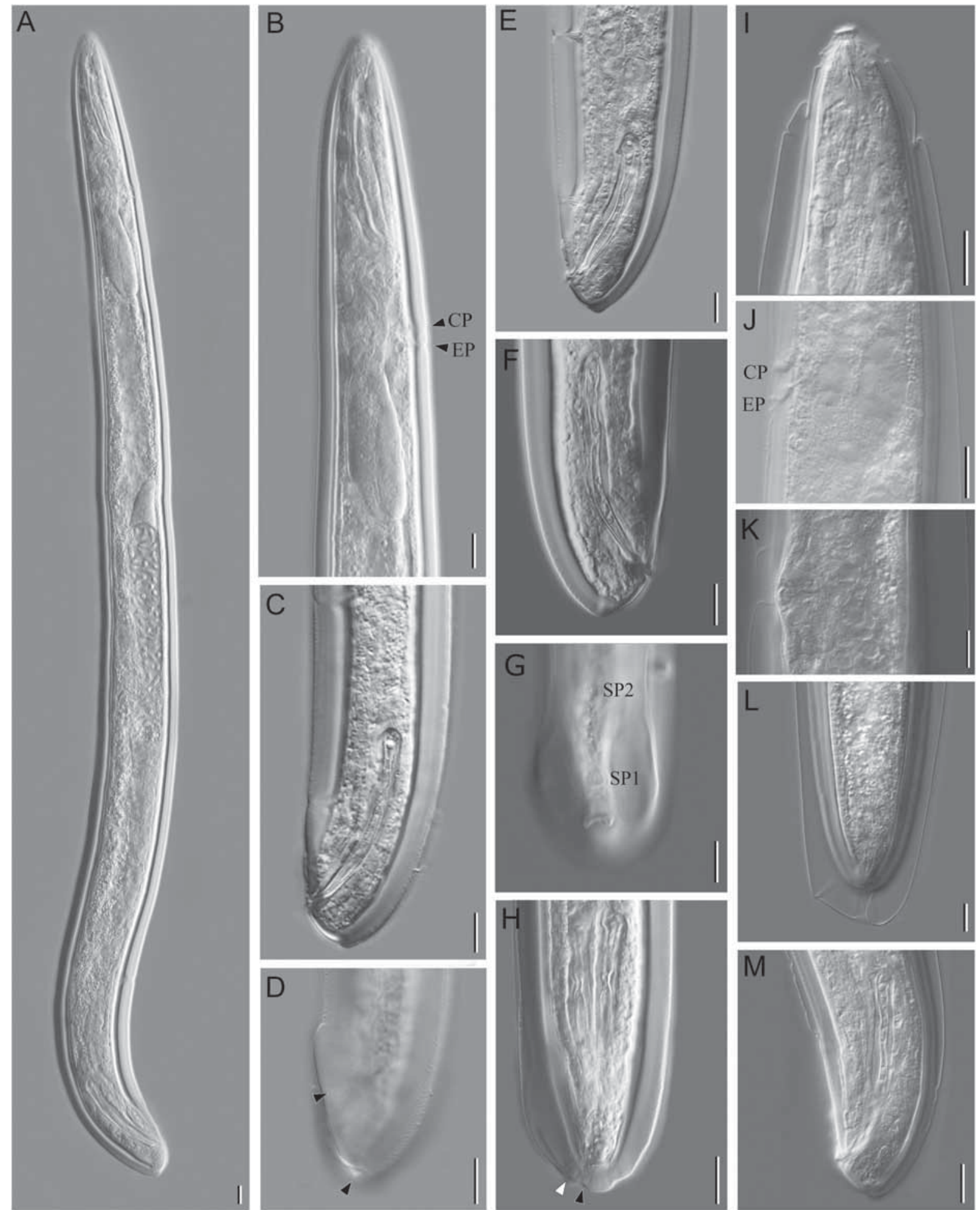

Figure 3. Light micrographs of Paratrichodorus hispanus. A-H, male topotypes. A, total view. B, neck region. C, E, posterior body region showing three precloacal supplements and copulatory apparatus. D, bursa. F, copulatory apparatus. G, posterior body region in ventral view. $\mathrm{H}$, posterior body region in ventral view, with arrow pointing to caudal pore. I, K, $\mathrm{L}$, female paratypes. I, anterior body region showing swollen body cuticle. K, vaginal region. L, posterior body region. J, M, male paratypes. J, presence of one ventromedian cervical papilla shortly anterior to secretory-excretory pore in neck region. M, posterior body region with copulatory apparatus. Abbreviations: CP, cervical papillae; EP, excretory pore; SP1, medioventral precloacal supplement 1; SP2, medioventral precloacal supplement 2. Scale bars: $10 \mu \mathrm{m}$.

(ㄷ) 2018 The Linnean Society of London, Zoological Journal of the Linnean Society, 2019, 185, 656-692 

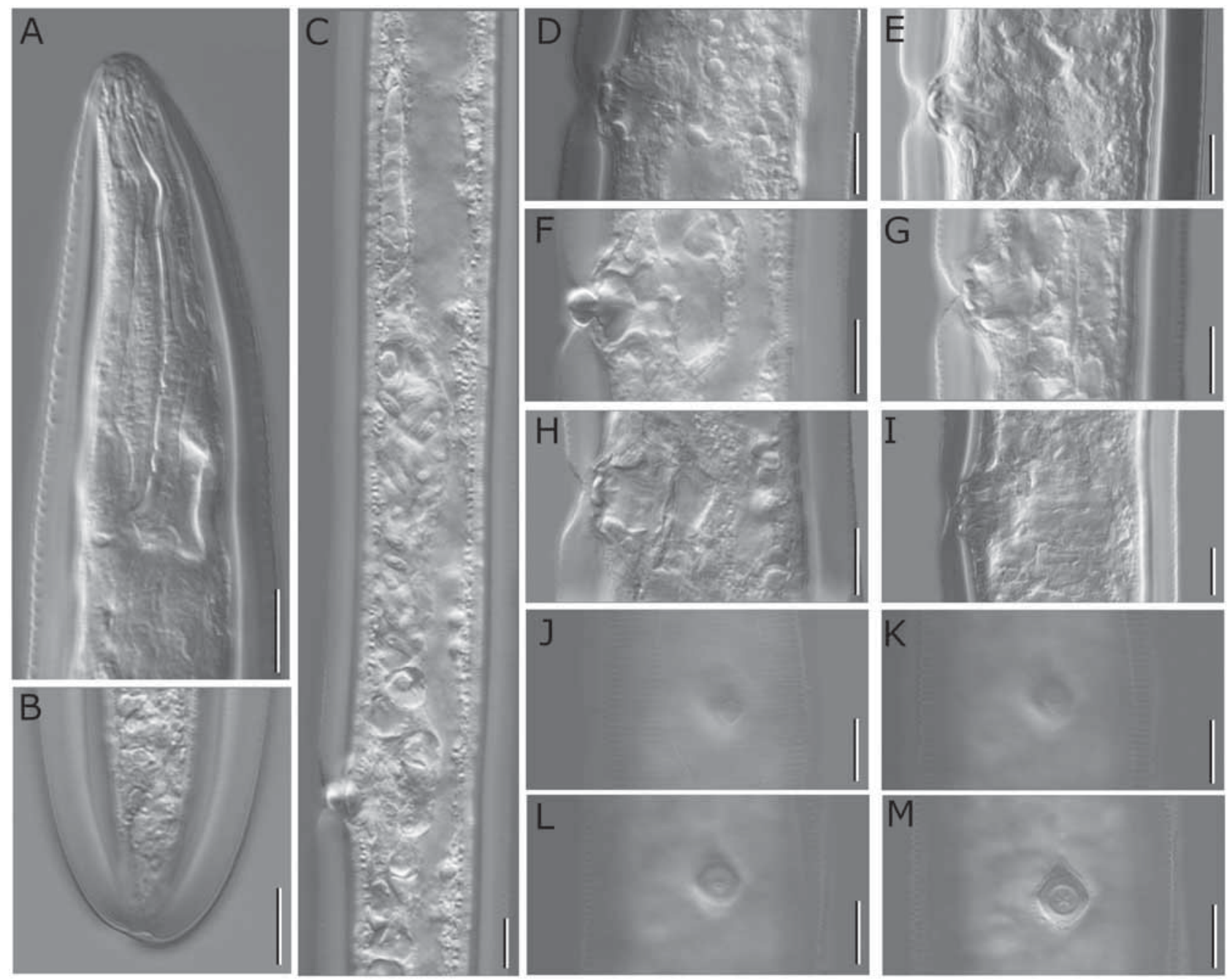

Figure 4. Light micrographs of Paratrichodorus hispanus. Female topotypes. A, onchiostyle region showing impact of fixation. B, tail region. $\mathrm{C}-\mathrm{M}$, vaginal region. $\mathrm{C}$, anterior genital branch with sperm and vagina with copulatory plug. F, vagina with copulatory plug. J-M, optical transverse section of vulva-vagina in ventral view. J, surface view. K, just below surface, a transverse slit. L, pore-like at level tip sclerotized pieces. M, star-like lumen posterior to sclerotized pieces. Scale bars: $10 \mu \mathrm{m}$.

is large sausage-shaped, average $7.0 \mu \mathrm{m} \times 2.0 \mu \mathrm{m}$ in size. Spicules were similar to the paratype, with a narrow undulated calomus $1.0-2.0 \mu \mathrm{m}$ wide and maximal width of blade 2.5-3.0 $\mu \mathrm{m}$, showing some slight variation in width and undulation attributable to fixation. Bursa short, rounded, extending from level SP2 to immeditately anterior to the pair of postcloacal supplements (PSP); dorsal anal flap bifid (Fig. 3). Tail similar to paratype, about half as long as anal body width; body cuticle dorsally thickened until the terminal end, which may appear slightly knob-like, swollen, $4.0 \mu \mathrm{m}$ thick (average); ventrally, tail cuticle thin $(2.0-2.5 \mu \mathrm{m})$. Postcloacal supplements subterminal, with ventral cuticle between PSP and cloacal opening much thinner; pair of caudal pores slightly dorsal to PSP.
Despite the smaller measurements, largely attributable to the impact of fixation, the topotypes fit the type description. For the first time, well-structured secretion plugs have been observed in several females in a species of Paratrichodorus. Sturhan (1985) illustrated and described the presence of a secretory plug in the vagina, albeit without a clear structure. The plug shape is different from the plugs observed in Trichodorus species, but most closely resembles the bifid plug in Trichodorus elefjohnsoni Bernard, 1992, albeit much smaller (Decraemer, 2012).

Specific alphanumeric codes (in parentheses are exceptions) of the polytomous key adapted from Decraemer \& Baujard (1998): (1) for males = presented with topotypes between [] when different from type specimens, A213 [A112] (average, minimum, 


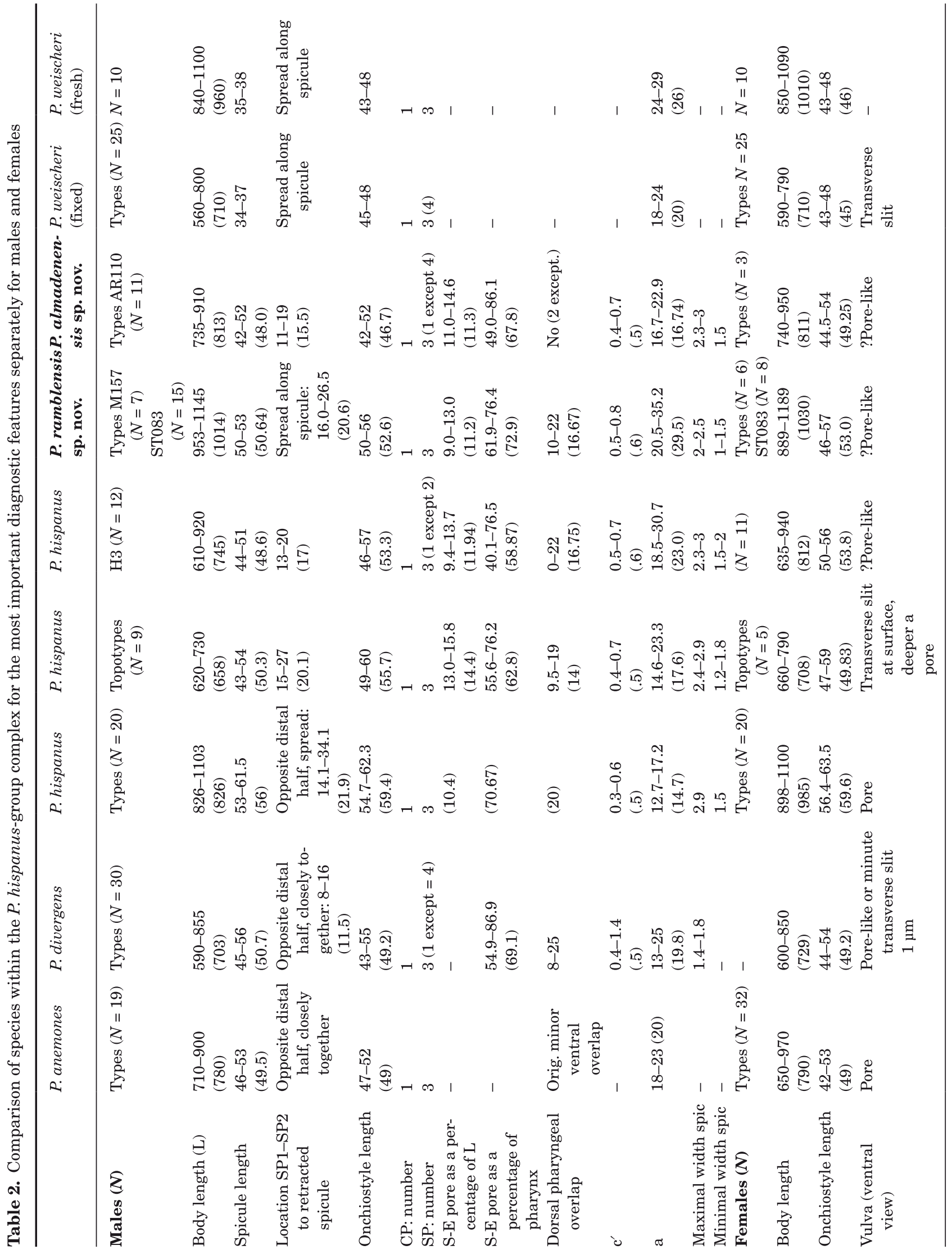




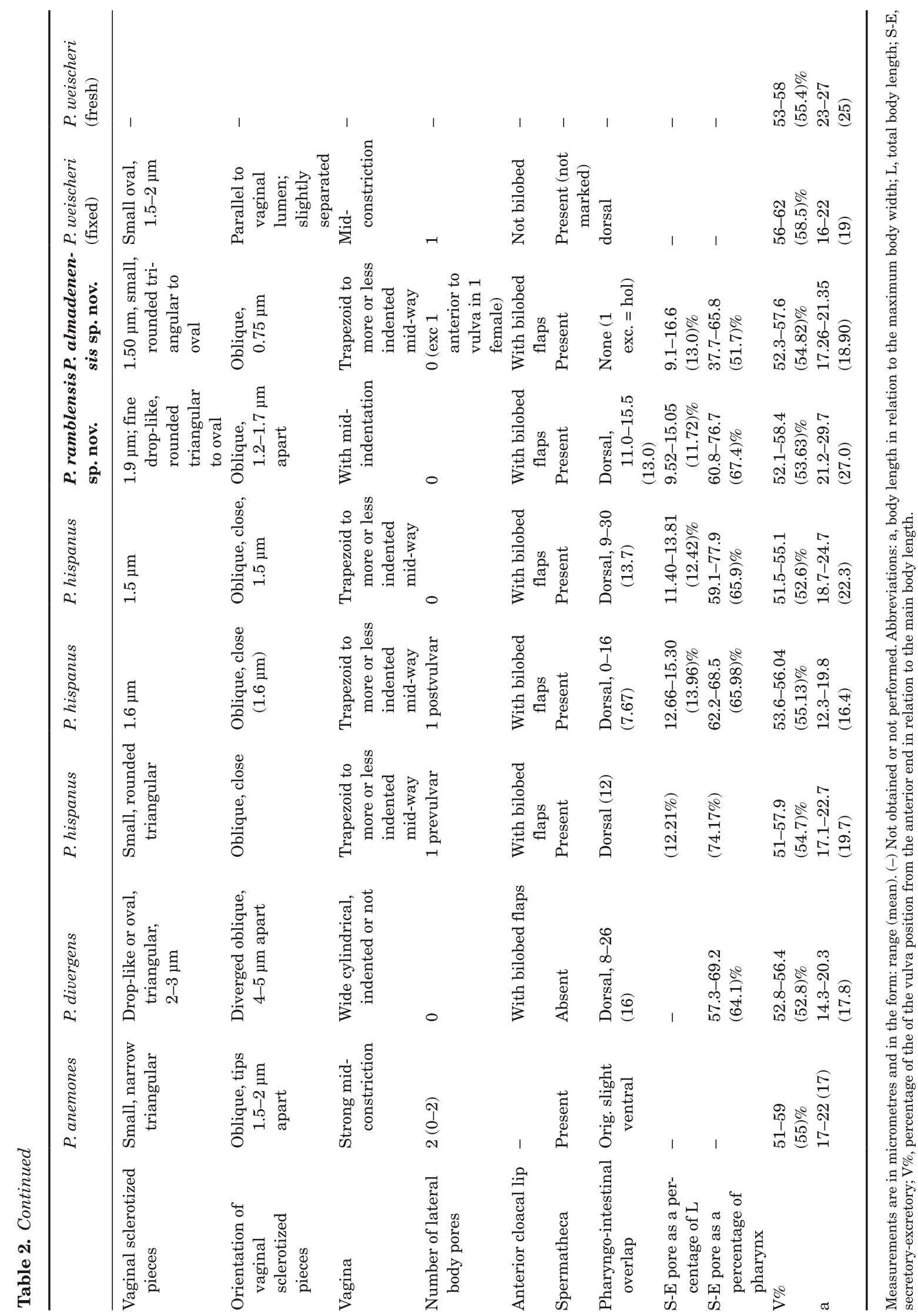


Table 3. Morphometrics of Paratrichodorus hispanus Roca \& Arias, 1986 and new topotype material from wheat at Santa Olalla (Toledo, Spain)

\begin{tabular}{|c|c|c|c|c|c|}
\hline & $\begin{array}{l}\text { Holotype } \\
\text { male }\end{array}$ & $\begin{array}{l}\text { Paratype } \\
\text { males }\end{array}$ & $\begin{array}{l}\text { Paratype } \\
\text { females }\end{array}$ & $\begin{array}{l}\text { Topotype } \\
\text { males }\end{array}$ & $\begin{array}{l}\text { Topotype } \\
\text { females }\end{array}$ \\
\hline$N$ & & $20(1)$ & $20(1)$ & 9 & 5 \\
\hline $\mathrm{L}$ & 976 & $\begin{array}{l}826-1103 \\
956 \pm 85(1015)\end{array}$ & $\begin{array}{l}898-1100 \\
985 \pm 57(920)\end{array}$ & $\begin{array}{l}620-730 \\
658 \pm 29.8\end{array}$ & $\begin{array}{l}660-790 \\
708 \pm 51.1\end{array}$ \\
\hline Onchiostyle & 58.0 & $\begin{array}{l}55.0-62.0 \\
59.4 \pm 2.0(58)\end{array}$ & $\begin{array}{l}56.5-63.5 \\
59.6 \pm 2.0(56.5)\end{array}$ & $\begin{array}{l}49.0-60.0 \\
55.7 \pm 3.53\end{array}$ & $\begin{array}{l}47.0-59.0 \\
49.83 \pm 4.46\end{array}$ \\
\hline Onchium & - & $(31.0)$ & $(29.0)$ & $\begin{array}{l}24.0-31.0 \\
28.4 \pm 1.9\end{array}$ & $\begin{array}{l}24.0-32.0 \\
28 \pm 2.5\end{array}$ \\
\hline Pharyngostom & - & $(65.0)$ & $(63.0)$ & $\begin{array}{l}58.0-66.0 \\
63.1 \pm 2.9\end{array}$ & $\begin{array}{l}61-66 \\
63.0 \pm 1.8\end{array}$ \\
\hline Pharynx (along)/neck (original) & - & $\begin{array}{l}128.8-174.8(142) \\
148.7 \pm 10.1(150)\end{array}$ & $\begin{array}{l}133.3-170.3 \\
158.09 \pm 10.0(151)\end{array}$ & $\begin{array}{l}129-169 \\
153 \pm 11.6\end{array}$ & $\begin{array}{l}146-155 \\
149.2 \pm 3.1\end{array}$ \\
\hline Dorsal pharyngeal overlap & - & $(20)$ & (12) & $\begin{array}{l}9.5-19.0 \\
14 \pm 3.4\end{array}$ & $\begin{array}{l}0-23 \\
12.6 \pm 8.11\end{array}$ \\
\hline Anterior to guide ring & 23.5 & $\begin{array}{l}23.5-28.0 \\
25.6 \pm 1.3(22.5)\end{array}$ & $\begin{array}{l}22.0-29.0 \\
25.3 \pm 1.5(23.5)\end{array}$ & $\begin{array}{l}14.0-27.0 \\
22.4 \pm 3.5\end{array}$ & $\begin{array}{l}21.5-25.0 \\
23.3 \pm 1.2\end{array}$ \\
\hline Anterior to CP1 & - & $(100)$ & - & $\begin{array}{l}82.0-95.0 \\
88.4 \pm 5.44\end{array}$ & - \\
\hline Anterior to S-E pore & 109.5 & $\begin{array}{l}97.5-122.0 \\
110.5 \pm 6.2(106)\end{array}$ & $\begin{array}{l}103.0-123.5 \\
114.0 \pm 5.0(112)\end{array}$ & $\begin{array}{l}84.0-103.0 \\
94.0 \pm 6.2\end{array}$ & $\begin{array}{l}92.0-101.0 \\
98.4 \pm 3.3\end{array}$ \\
\hline CP1 to S-E pore & - & $\begin{array}{l}4.7-20 \\
9.7 \pm 3.3 \\
(6)\end{array}$ & - & $\begin{array}{l}3-11 \\
6.1 \pm 2.4\end{array}$ & - \\
\hline Anterior to LP & - & $(88)$ & - & $\begin{array}{l}70-89 \\
80.3 \pm 8.0\end{array}$ & - \\
\hline mbd at cardia & - & $(39.5)$ & $(49.0)$ & $\begin{array}{l}35.0-45.0 \\
39.2 \pm 3.4\end{array}$ & $\begin{array}{l}35.0-46.0 \\
39.1 \pm 3.7\end{array}$ \\
\hline $\mathrm{mbd}$ at mid-body/vulva & - & $\begin{array}{l}41.0-53.0 \\
47.4 \pm 2.6\end{array}$ & $(59.0)$ & $\begin{array}{l}37.0-49.0 \\
45.4 \pm 5.4\end{array}$ & $\begin{array}{l}38.0-54.0 \\
44.0 \pm 5.7\end{array}$ \\
\hline $\mathrm{abd} / \mathrm{Q}$ : length of anterior ovary & - & $(22.0)$ & $(54.0)$ & $\begin{array}{l}21.0-22.5 \\
21.0 \pm 3.4\end{array}$ & $\begin{array}{l}31.0-71.0 \\
47.2 \pm 14.9\end{array}$ \\
\hline $\begin{array}{l}\text { Spicule length/o: length } \\
\text { of anterior branch }\end{array}$ & 55.8 & $\begin{array}{l}53.0-61.7 \\
56.0 \pm 2.8(51)\end{array}$ & $(200)$ & $\begin{array}{l}43.0-54.0 \\
50.3 \pm 2.5\end{array}$ & $\begin{array}{l}144-217 \\
190.6 \pm 25.2\end{array}$ \\
\hline $\begin{array}{l}\text { Gubernaculum/q: length } \\
\text { of posterior ovary }\end{array}$ & 14.7 & $\begin{array}{l}13.5-16.4 \\
14.5 \pm 0.8(12)\end{array}$ & $(54)$ & $\begin{array}{l}14.0-19.0 \\
15.9 \pm 2.0\end{array}$ & $\begin{array}{l}40.0-65.0 \\
56.4 \pm 8.7\end{array}$ \\
\hline $\begin{array}{l}\text { Distance SP1-anus/Q: } \\
\text { length of posterior branch }\end{array}$ & 11.1 & $\begin{array}{l}7.6-12.9 \\
10.6 \pm 1.6(12)\end{array}$ & $(203)$ & $\begin{array}{l}10.5-12.5 \\
11.6 \pm 0.8\end{array}$ & $\begin{array}{l}158-224 \\
192.8 \pm 25.3\end{array}$ \\
\hline $\begin{array}{l}\text { Distance SP1-SP2/P: } \\
\text { length of vagina }\end{array}$ & 16.9 & $\begin{array}{l}14.1-34.1 \\
21.9 \pm 4.46(17.5)\end{array}$ & (11) & $\begin{array}{l}15-27 \\
20.1 \pm 3.6\end{array}$ & $\begin{array}{l}10.5-15 \\
12.7 \pm 1.6\end{array}$ \\
\hline $\begin{array}{l}\text { Distance SP2-SP3/Q: } \\
\text { vagina as a percentage of cbw }\end{array}$ & 90.0 & $\begin{array}{l}78.2-144 \\
100.1 \pm 18.2(125)\end{array}$ & $(18.6)$ & $\begin{array}{l}54.0-84.0 \\
69.4 \pm 9.3\end{array}$ & $\begin{array}{l}26.0-33.0 \\
29.0 \pm 2.5\end{array}$ \\
\hline Tail/o: G1 & - & $(10.5)$ & $(21.8)$ & $\begin{array}{l}8.5-12.0 \\
10.2 \pm 1.03\end{array}$ & $\begin{array}{l}21.0-32.5 \\
27.1 \pm 4.3\end{array}$ \\
\hline $\mathrm{a}$ & 20.5 & $\begin{array}{l}13-25 \\
19.8 \pm 2.6\end{array}$ & $\begin{array}{l}17.1-22.7 \\
19.7 \pm 1.5(15.5)\end{array}$ & $\begin{array}{l}12.7-17.2 \\
14.7 \pm 1.5\end{array}$ & $\begin{array}{l}12.3-19.8 \\
16.4 \pm 2.9\end{array}$ \\
\hline $\mathrm{b}$ & 6.5 & $\begin{array}{l}5.6-7.7 \\
6.4 \pm 0.6(7.1)\end{array}$ & $\begin{array}{l}5.4-7.3 \\
6.2 \pm 0.5\end{array}$ & $\begin{array}{l}4.0-4.9 \\
4.4 \pm 0.4\end{array}$ & $\begin{array}{l}4.3-5.4 \\
4.8 \pm 0.4\end{array}$ \\
\hline$c / Q: V$ & 83.4 & $\begin{array}{l}55.8-102 \\
77.8 \pm 13.9\end{array}$ & $\begin{array}{l}51.0-57.9 \\
54.7 \pm 1.8(6.1)\end{array}$ & - & $\begin{array}{l}53.6-56.04 \\
55.1 \pm 0.8\end{array}$ \\
\hline T/Q: G2 & 65.0 & $\begin{array}{l}60-73 \\
67.4 \pm 3.5(62.9)\end{array}$ & - & $\begin{array}{l}59.4-66.3 \\
63.3 \pm 2.7\end{array}$ & $\begin{array}{l}23.2-32.0 \\
22.2 \pm 2.9\end{array}$ \\
\hline
\end{tabular}


Table 3. Continued

\begin{tabular}{llllll}
\hline & $\begin{array}{l}\text { Holotype } \\
\text { male }\end{array}$ & $\begin{array}{l}\text { Paratype } \\
\text { males }\end{array}$ & $\begin{array}{l}\text { Paratype } \\
\text { females }\end{array}$ & $\begin{array}{l}\text { Topotype } \\
\text { males }\end{array}$ & $\begin{array}{l}\text { Topotype } \\
\text { females }\end{array}$ \\
\hline $\mathrm{c}^{\prime}$ & 0.5 & $0.3-0.6$ & - & $0.4-0.7$ & - \\
& & $0.5 \pm 0.1(0.4)$ & & $0.5 \pm 0.1$ & $13.0-15.8$ \\
S-E pore as a percentage & - & $(10.4)$ & $(12.2)$ & $14.4 \pm 0.8$ & $14.7-15.3$ \\
$\quad \begin{array}{l}\text { of L from anterior end } \\
\text { S-E pore as a percentage }\end{array}$ & - & $(70.7)$ & $(74.2)$ & $55.6-76.2$ & $62.2-68.5$ \\
$\quad$ of pharynx from anterior end & & & & $62.8 \pm 7.3$ & $66.0 \pm 2.5$ \\
\hline
\end{tabular}

Measurements are in micrometres and in the form: range and mean $\pm \mathrm{SD}$; values of re-measured paratype males and females are given in parentheses. Abbreviations: a body length/maximal body width; abw (anal body width); b body length/pharyngeal length; $c$ body length/tail length; $c^{\prime}$, tail length/ body width at anus; cbw, cloacal body width; CP, ventromedian cervical papilla; CP1, anterior ventromedian cervical papilla; G1 and G2, (anterior and posterior gonad length, respectively/body length) $\times 100 ; \mathrm{L}$, (total body length); LP, labial papilla; mbw, (maximal body width; $N$, number of specimens studied; S-E pore, excretory pore; SP1, SP2 and SP3, posterior, second and third precloacal supplements, respectively; T, (distance from cloacal aperture to anterior end of testis/body length) $\times 100 ; \mathrm{V}$, (distance from anterior end to vulva/body length) $\times 100$

maximum), B23 [B22], C23 [C22], D11, E0, F3, G22, H33 (2), I33, J200, K33, L88, M270, N11, O100, P1; and (2) for females presented with topotypes between [] when different from type specimens, A213 [A212], B23 [B22], C1, D1, E30, F30, G1, H88 [H86 (3)], I11, J11, K30 [K23], L11 [L12], M1, N1, O11, P11, Q1, R22, $\mathrm{S} 1, \mathrm{~T} 1$.

\section{Other populations (Figs 5, 6; Tables 2, 4)}

Several populations of this species were collected from the rhizosphere of cultivated olive at Hinojos (H0003), Huelva province, southern Spain, the rhizosphere of wild olive (AR099, AR100), stone pine and cork oak (H0027) at El Rocio, Huelva (Spain), grapevine (388GR) at Bollullos par del Condado (Huelva, Spain), cork oak (HATR) at Villamanrique de la Condesa (Sevilla, Spain), eucalyptus (H0169) at Almonte (Huelva, Spain), stone pine (H0179) and eucalyptus (H0169) at Lucena del Puerto (Huelva, Spain), and wild olive (AR139) at Aroche (Huelva, Spain) and sample AR0140 at Rosal de la Frontera (Huelva, Spain).

Description of male: Body appearance straight, medium-sized body (average $745 \mu \mathrm{m}$, in type specimens). Onchiostyle medium-sized (average $53 \mu \mathrm{m}$, in type specimens) with onchium about half as long; pharynx with gland nuclei (dorsal and posterior ventrosublateral pair) clearly separated and with developed dorsal intestinal overlap ( 17 $\mu \mathrm{m}$ average). One ventromedian cervical papilla shortly $(6.5 \mu \mathrm{m}$ average) anterior to S-E pore opposite mid-pharyngeal bulb. Lateral body pore at level of S-E pore in holotype, but in most paratypes shortly anterior to CP. Males monorchic with testes on average $133 \mu \mathrm{m}$ long and sausage-shaped sperm nucleus $6.0 \mu \mathrm{m} \times 2.0 \mu \mathrm{m}$ in size. Medium-sized spicules (average $48.5 \mu \mathrm{m}$, in type specimens) with an irregular undulated (kinking) calomus (1.5-2.0 $\mu \mathrm{m}$ wide), and blade maximum width
$2.5 \mu \mathrm{m}$. Three precloacal supplements (one exception with two, i.e. no SP2), of which two are situated within the retracted spicules, with SP2 at mid-spicule level; a pair of subterminal postcloacal supplements adjacent to a pair of caudal pores. Tail half the anal body width in length.

Description of female: Females largely similar to males. Sperm stored in a spermatheca near the oviduct; vagina ( $27 \%$ on average of corresponding body width), with variable degree of contraction of vaginal constrictor muscles, but usually vagina clearly constricted mid-way. Vaginal sclerotized pieces small (1.5 $\mu \mathrm{m}$ on average), rounded triangular to rarely round, obliquely oriented with tips close to very close; no lateral body pores observed. Secretory plug regularly present in vagina and similar in shape to that in $P$. hispanus topotype females and observed in both other populations; rarely, plug in uterus.

Remarks: Apart from the absence of sublateral body pores in females of these populations of $P$. hispanus (code Q), no other morphological difference was observed from $P$. hispanus, especially when topotype specimens (see code) were examined. This could be largely attribugtable to the great impact of fixation, resulting in a wide range in certain morphometrics. However, the H18 population, with its smaller onchiostyle ( $44 \mu \mathrm{m}$ on average vs. $53 \mu \mathrm{m}$ in H0003 and $56 \mu \mathrm{m}$ in AR100) and smaller (average $40 \mu \mathrm{m}$ vs. $48.5 \mu \mathrm{m}$ in $\mathrm{H} 3$ and $52 \mu \mathrm{m}$ in AR100), more slender spicules (calomus width $1.5-2.0 \mu \mathrm{m}$ and blade maximum $2.5 \mu \mathrm{m}$, whereas in AR100 males, calomus 1.5-2.0 $\mu \mathrm{m}$ wide and blade 2.5-3.0 $\mu \mathrm{m}$ ), appears slightly different morphologically but not molecularly. Intraspecific variability was also observed within the $\mathrm{H} 3$ population in the degree of anterior-dorsal intestinal overlap over the pharynx, hardly present in the holotype but $8-22 \mu \mathrm{m}$ long in male paratypes. 
Table 4. Morphometrics of Paratrichodorus hispanus Roca \& Arias, 1986 males from cultivated and wild olive at Hinojos and El Rocío (Huelva, Spain)

\begin{tabular}{|c|c|c|c|c|c|c|}
\hline & $\begin{array}{l}\text { Males (H3) } \\
\text { Hinojos, } \\
\text { cultivated olive }\end{array}$ & $\begin{array}{l}\text { Males (AR100) } \\
\text { El Rocío, wild } \\
\text { olive }\end{array}$ & $\begin{array}{l}\text { Males (H18) } \\
\text { Hinojos, } \\
\text { cultivated olive }\end{array}$ & $\begin{array}{l}\text { Females (H3) } \\
\text { Hinojos, } \\
\text { cultivated } \\
\text { olive }\end{array}$ & $\begin{array}{l}\text { Females } \\
\text { (AR100) } \\
\text { El Rocío, } \\
\text { wild olive }\end{array}$ & $\begin{array}{l}\text { Female (H18) } \\
\text { Hinojos, } \\
\text { cultivated } \\
\text { olive }\end{array}$ \\
\hline$N$ & 12 & 11 & 4 & 11 & 8 & 1 \\
\hline \multirow[t]{2}{*}{$\mathrm{L}$} & $610-920$ & $715-945$ & $620-820$ & $635-940$ & $685-1035$ & 580 \\
\hline & $745 \pm 86$ & $836 \pm 61$ & $710 \pm 88$ & $812 \pm 84$ & $901 \pm 110$ & \\
\hline \multirow[t]{2}{*}{ Onchiostyle } & $46.0-57.0$ & $46.0-64.0$ & $41.0-46.0$ & $50.0-56.0$ & $49.0-65.0$ & 49.0 \\
\hline & $53.3 \pm 3.2$ & $56.3 \pm 6.7$ & $44.1 \pm 1.9$ & $53.8 \pm 2.9$ & $59 \pm 5.7$ & \\
\hline \multirow[t]{2}{*}{ Onchium } & $22.0-32.0$ & $21.0-34.0$ & $20.0-24.0$ & $26-29$ & $20-35$ & 26.0 \\
\hline & $28.0 \pm 2.3$ & $27.7 \pm 5.7$ & $22.3 \pm 1.8$ & $27.7 \pm 1.0$ & $29.8 \pm 4.8$ & \\
\hline \multirow[t]{2}{*}{ Pharyngostom } & $50.0-69.0$ & $46.0-78.0$ & $47.0-54.0$ & $59-68$ & $65-75$ & 56.0 \\
\hline & $61.6 \pm 5.4$ & $67.4 \pm 9.9$ & $51.8 \pm 2.9$ & $63.7 \pm 2.7$ & $67.8 \pm 7.1$ & \\
\hline \multirow[t]{2}{*}{ Pharynx (along) } & $136-166$ & $112-198$ & $117-133$ & $131-164$ & $160-188$ & 129.0 \\
\hline & $152.0 \pm 9$ & $161.9 \pm 29.0$ & $127.0 \pm 6.4$ & $153 \pm 10$ & $178 \pm 12.8$ & \\
\hline \multirow{2}{*}{$\begin{array}{l}\text { Dorsal pharyngeal } \\
\text { overlap }\end{array}$} & $0.0-22.0$ & $14.0-31.0$ & $0.0-12.0$ & $9.0-30.0$ & $9.0-31.0$ & - \\
\hline & $16.8 \pm 5.2$ & $22.40 \pm 7.17$ & $6.0 \pm 6.0$ & $13.7 \pm 7.32$ & $19.8 \pm 8.3$ & \\
\hline \multirow{2}{*}{$\begin{array}{l}\text { Anterior to } \\
\text { guide ring }\end{array}$} & $18.5-27.0$ & $15.0-30.0$ & - & $19.0-27.0$ & $19.0-28.0$ & 22.0 \\
\hline & $23.9 \pm 2.2$ & $23.7 \pm 4.8$ & & $22.9 \pm 2.5$ & $25.6 \pm 3.1$ & \\
\hline \multirow{2}{*}{$\begin{array}{l}\text { Anterior to } \\
\text { nerve ring }\end{array}$} & $68.0-71.0$ & $46.0-80.0$ & - & $69.0-83.0$ & $79.0-80.0$ & 61.0 \\
\hline & $67.3 \pm 3.3$ & 67.7 & & $74.6 \pm 5.4$ & $79.7 \pm 0.5$ & \\
\hline \multirow[t]{2}{*}{ Anterior to $\mathrm{CP} 1$} & $53.0-99.0$ & $90.0-116.0$ & $62.0-79.0$ & - & - & - \\
\hline & $82.5 \pm 13.1$ & $101.7 \pm 8.0$ & $74.3 \pm 7.1$ & & & \\
\hline $\begin{array}{l}\text { Distance } \\
\text { CP1_S-E nore }\end{array}$ & $5.0-12.0$ & $2.0-8.5$ & $3.0-10.0$ & - & - & - \\
\hline CP1-S-E pore & $6.4 \pm 2.0$ & $5.8 \pm 2.3$ & $5.3 \pm 2.9$ & & & \\
\hline \multirow{2}{*}{$\begin{array}{l}\text { Anterior to } \\
\text { S-E pore }\end{array}$} & $59-104$ & $93-124$ & $65-89$ & $88.0-108.0$ & $82.0-132.0$ & 88.0 \\
\hline & $88.9 \pm 12.1$ & $107.9 \pm 9.7$ & $79.5 \pm 9.0$ & $100.3 \pm 5.4$ & $114 \pm 13.8$ & \\
\hline \multirow[t]{2}{*}{ Anterior to LP } & $62-87$ & $81-109$ & - & - & - & - \\
\hline & $77.5 \pm 8.7$ & $99.7 \pm 10.0$ & & & & \\
\hline \multirow[t]{2}{*}{ mbd at cardia } & $26.0-38.0$ & $32.0-55.0$ & $23.0-29.0$ & $30.0-37.0$ & $35.0-48.0$ & 24.0 \\
\hline & $31.0 \pm 3.4$ & $41.9 \pm 8.0$ & $24.9 \pm 2.5$ & $33.7 \pm 2.3$ & $41.7 \pm 5.2$ & \\
\hline \multirow[t]{2}{*}{ mbd mid-body } & $28.0-39.0$ & $36.0-61.0$ & $26.0-32.5$ & $32.0-42.0$ & $40.0-63.0$ & 26.0 \\
\hline & $32.9 \pm 3.6$ & $48.9 \pm 7.6$ & $24.9 \pm 2.8$ & $37.0 \pm 4.2$ & $50 \pm 6.1$ & \\
\hline \multirow{2}{*}{$\begin{array}{l}\text { Length of } \\
\text { anterior ovary }\end{array}$} & - & - & - & $31-115$ & $54-146$ & 34.0 \\
\hline & & & & $70.1 \pm 26.5$ & $91.8 \pm 43.3$ & \\
\hline \multirow{2}{*}{$\begin{array}{l}\text { Length of anterior } \\
\text { branch }\end{array}$} & - & - & - & $156-444$ & $162-433$ & 140.0 \\
\hline & & & & $250 \pm 65$ & $269.3 \pm 80.0$ & \\
\hline \multirow{2}{*}{$\begin{array}{l}\text { Length of posterior } \\
\text { ovary }\end{array}$} & - & - & - & $36.0-114.0$ & $46.0-129.0$ & 61.0 \\
\hline & & & & $69.4 \pm 33.3$ & $76.6 \pm 33.7$ & \\
\hline \multirow{2}{*}{$\begin{array}{l}\text { Length of posterior } \\
\text { branch }\end{array}$} & - & - & - & $126-319$ & $69-363$ & 188.0 \\
\hline & & & & $205 \pm 65$ & $237.8 \pm 106.2$ & \\
\hline \multirow[t]{2}{*}{ Length of vagina } & - & - & - & $7.0-12.0$ & $11.0-15.0$ & 11.0 \\
\hline & & & & $10.2 \pm 1.7$ & $12.5 \pm 1.2$ & \\
\hline \multirow{2}{*}{$\begin{array}{l}\text { Vagina as a } \\
\text { percentage of cbw }\end{array}$} & - & - & - & $18.4-36.4$ & $20.6-37.5$ & 42.2 \\
\hline & & & & $27.5 \pm 6.7$ & $25.7 \pm 5.4$ & \\
\hline \multirow[t]{2}{*}{ abd } & $16.0-21.0$ & $18.0-27.0$ & $14.0-18.0$ & - & - & - \\
\hline & $18.8 \pm 1.5$ & $22.1 \pm 3.6$ & $15.5 \pm 1.7$ & & & \\
\hline Spicule length & $44.0-51.0$ & $42.0-58.0$ & $37.0-43.0$ & - & - & - \\
\hline & $48.6 \pm 2.7$ & $52.1 \pm 6.3$ & $39.88 \pm 2.5$ & & & \\
\hline Gubernaculum & $12.0-17.0$ & $11.5-17.0$ & $13.0-14.5$ & - & - & - \\
\hline & $14.3 \pm 3.2$ & $14.1 \pm 2.7$ & $13.8 \pm 0.6$ & & & \\
\hline Distance SP1-cloacal & $6.0-12.0$ & $7.5-14.0$ & $5.0-8.5$ & - & - & - \\
\hline opening & $9.8 \pm 2.1$ & $11.4 \pm 2.6$ & $7.0 \pm 1.4$ & & & \\
\hline
\end{tabular}


Table 4. Continued

\begin{tabular}{|c|c|c|c|c|c|c|}
\hline & $\begin{array}{l}\text { Males (H3) } \\
\text { Hinojos, } \\
\text { cultivated olive }\end{array}$ & $\begin{array}{l}\text { Males (AR100) } \\
\text { El Rocío, wild } \\
\text { olive }\end{array}$ & $\begin{array}{l}\text { Males (H18) } \\
\text { Hinojos, } \\
\text { cultivated olive }\end{array}$ & $\begin{array}{l}\text { Females }(\mathrm{H} 3) \\
\text { Hinojos, } \\
\text { cultivated } \\
\text { olive }\end{array}$ & $\begin{array}{l}\text { Females } \\
\text { (AR100) } \\
\text { El Rocío, } \\
\text { wild olive }\end{array}$ & $\begin{array}{l}\text { Female (H18) } \\
\text { Hinojos, } \\
\text { cultivated } \\
\text { olive }\end{array}$ \\
\hline \multirow[t]{2}{*}{ Distance SP1-SP2 } & $13.0-20.0$ & $11.0-29.0$ & $12.0-18.0$ & - & - & - \\
\hline & $17.9 \pm 2.1$ & $18.6 \pm 5.6$ & $15.0 \pm 2.2$ & & & \\
\hline \multirow[t]{2}{*}{ Distance SP2-SP3 } & $51.0-144.0$ & $41.0-113.0$ & $53.0-72.0$ & - & - & - \\
\hline & $81.1 \pm 23.7$ & $78.2 \pm 22.7$ & $61.0 \pm 7.0$ & & & \\
\hline \multirow[t]{2}{*}{ Tail } & $8.5-14.0$ & $8.5-12.5$ & $9.0-11.0$ & - & - & - \\
\hline & $10.7 \pm 1.6$ & $11.7 \pm 2.1$ & $10.25 \pm 0.8$ & & & \\
\hline \multirow[t]{2}{*}{$\mathrm{a}$} & $18.5-30.7$ & $14.6-23.3$ & $23.92-29.73$ & $18.7-24.7$ & $14.0-21.6$ & 11.3 \\
\hline & $23.0 \pm 3.6$ & $17.6 \pm 2.6$ & $25.7 \pm 2.4$ & $22.3 \pm 2.3$ & $18.2 \pm 2.4$ & \\
\hline \multirow[t]{2}{*}{$\mathrm{b}$} & $4.0-6.8$ & $4.0-6.8$ & $4.7-6.6$ & $4.7-6.3$ & $3.8-5.6$ & 4.5 \\
\hline & $4.9 \pm 0.7$ & $5.3 \pm 1.0$ & $5.6 \pm 0.8$ & $5.5 \pm 1.5$ & $5.1 \pm 0.7$ & \\
\hline \multirow[t]{2}{*}{ c } & $62.0-84.6$ & $49.2-85.8$ & $62.2-74.6$ & - & - & - \\
\hline & $70.3 \pm 9.4$ & $73.5 \pm 12.1$ & $69.12 \pm 4.4$ & & & \\
\hline \multirow[t]{2}{*}{ G1 } & - & - & - & $18.1-53.3$ & $19.4-45.0$ & 24.2 \\
\hline & & & & $30.9 \pm 8.9$ & $29.6 \pm 7.6$ & \\
\hline \multirow[t]{2}{*}{ G2 } & - & - & - & $16.0-36.2$ & $8.2-44.6$ & 32.5 \\
\hline & & & & $25.5 \pm 6.5$ & $26.0 \pm 10.5$ & \\
\hline \multirow[t]{2}{*}{$\mathrm{T} / \mathrm{V}$} & $59.7-71.7$ & $43.9-78.2$ & $57.9-62.6$ & $51.1-55.1$ & $53.4-65.9$ & 53.9 \\
\hline & $66.5 \pm 11.1$ & $64.4 \pm 9.3$ & $59.7 \pm 2.0$ & $52.6 \pm 1.5$ & $58.2 \pm 3.9$ & \\
\hline \multirow[t]{2}{*}{$c^{\prime}$} & $0.5-0.7$ & $0.3-0.9$ & $1.4-1.6$ & - & - & - \\
\hline & $0.6 \pm 0.1$ & $0.5 \pm 0.13$ & $1.5 \pm 0.1$ & & & \\
\hline \multirow{2}{*}{$\begin{array}{l}\text { S-E pore as a per- } \\
\text { centage of } L \text { from } \\
\text { anterior end }\end{array}$} & $9.4-13.6$ & $11.2-16.4$ & $8.4-14.3$ & $11.4-13.8$ & $8.5-16.0$ & 15.2 \\
\hline & $11.9 \pm 1.7$ & $13.0 \pm 1.4$ & $11.5 \pm 2.5$ & $12.4 \pm 0.9$ & $12.8 \pm 2.0$ & \\
\hline \multirow{2}{*}{$\begin{array}{l}\text { S-E pore as a per- } \\
\text { centage of pharynx } \\
\text { from anterior end }\end{array}$} & $40.1-76.5$ & $58.5-94.6$ & $55.6-70.6$ & $59.1-77.9$ & $47.67-75.0$ & 68.2 \\
\hline & $58.9 \pm 9.75$ & $69.1 \pm 10.8$ & $62.5 \pm 5.4$ & $65.9 \pm 5.95$ & $63.4 \pm 8.4$ & \\
\hline
\end{tabular}

Measurements are in micrometres and in the form: range and mean \pm SD.

Abbreviations: a, body length/maximal body width; abw, anal body width; $b$, body length/pharyngeal length; $c$, body length/tail length; $c^{\prime}$, tail length/ body width at anus; cbw, cloacal body width; CP, ventromedian cervical papilla; CP1, anterior ventromedian cervical papilla; G1 and G2, (anterior and posterior gonad length, respectively/body length) $\times 100 ; \mathrm{L}$, total body length; LP, labial papilla; mbw, maximal body width; $N$, number of specimens studied; S-E pore, excretory pore; SP1, SP2 and SP3, (posterior, second and third precloacal supplements, respectively; T, (distance from cloacal aperture to anterior end of testis/body length) $\times 100 ; \mathrm{V}$, (distance from anterior end to vulva/body length) $\times 100$

Furthermore, within the type population the variation in measurements was rather large and overlapped with the range known for $P$. hispanus when including topotypes. Several female specimens had a secretion plug in the vagina, and some showed less or more contraction of the vaginal constrictor muscle, resulting in different appearances of the vagina. Specific alphanumeric codes (in parentheses are exceptions) of the polytomous key adapted from Decraemer \& Baujard (1998) are as follows: (1) for females = A212, B22, C1, D1, E33, F33, G2 (1), H 86 (3), I1, J1 (2), K23, L2, M1, N1, O1, P 1, Q4, R2, S1, T1; and (2) for males = A212, B22, C22, D11, E0, F3 (2), G22, H33, I3 (1), J100, K33, L88, M270, N11, O100, P1.

The code for males is closer to that of $P$. hispanus; similar observations were made for females, with code Q4 (body pores) as the only difference detected.

\section{PARATRICHODORUS ALMADENENSIS SP. NOV.}

(Figs 7, 8; TABles 2, 5)

urn:lsid:zoobank.org:act:45356A52-CFF1-4342-8EA2D4F958C6EDAB

Holotype: Female was extracted from soil samples collected from the rhizosphere of wild olive at Almadén de la Plata, Sevilla province, southern Spain $\left(37^{\circ} 46^{\prime} 55.7^{\prime \prime} \mathrm{N}, 6^{\circ} 08^{\prime} 05.8^{\prime \prime} \mathrm{W}\right)$ by J. Martín Barbarroja and G. León Ropero, mounted in pure glycerine and deposited in the Nematode Collection of the Ghent University, Ghent, Belgium (slide number UGMD 104342).

Paratypes: Male and female paratypes extracted from soil samples collected from the rhizosphere of 


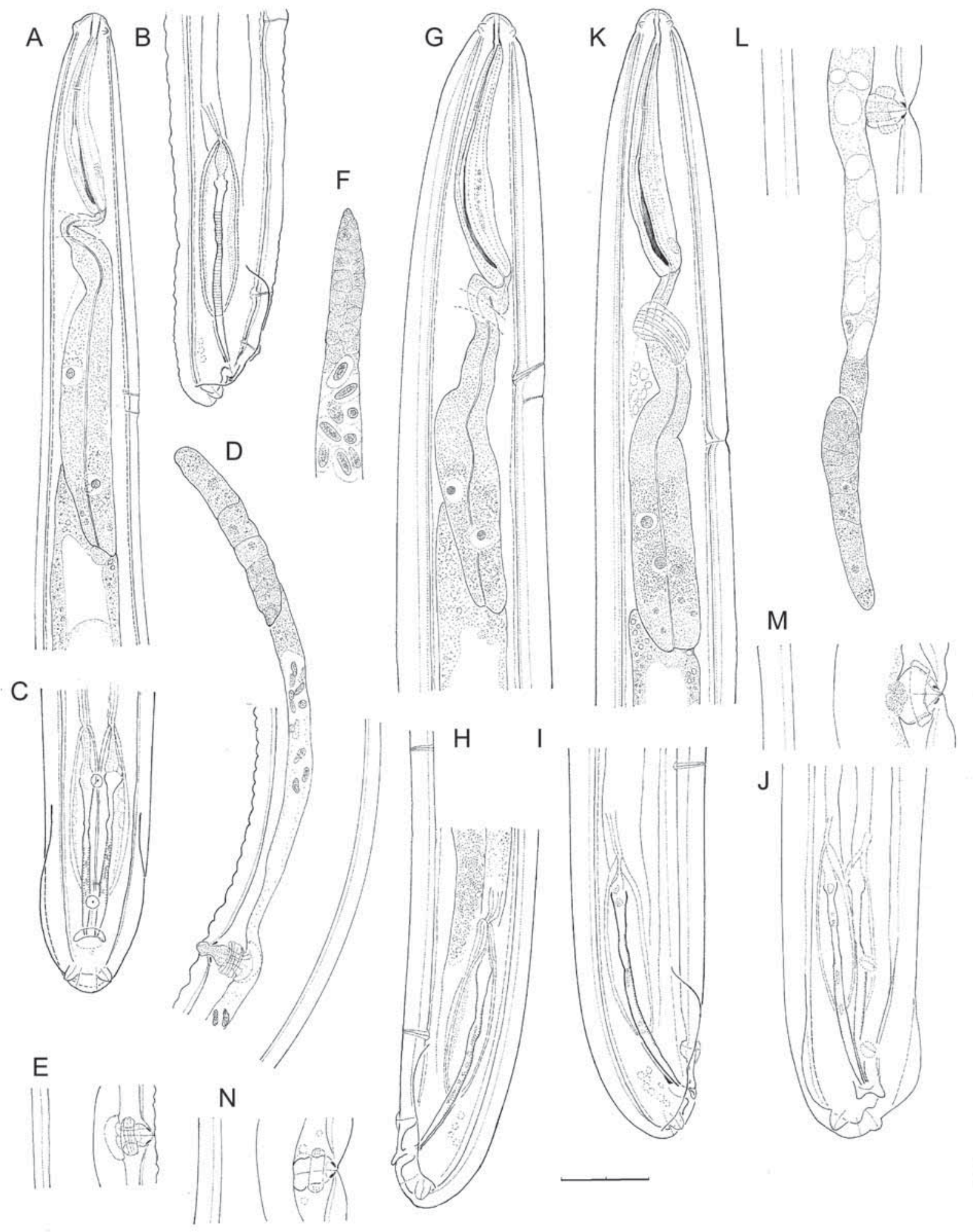

Figure 5. Line drawings of Paratrichodorus hispanus, other localities. A-C, F, males from H18. A, neck region. B, C, posterior body region with copulatory apparatus, with $\mathrm{C}$ in ventral view. F, anterior testis. D, E, N, females from H18. D, anterior genital branch and vagina with copulatory plug. E, N, vaginal region. G, H-J, males from H3. G, neck region. H-J, posterior body region and copulatory apparatus. J, oblique ventral view. K-M, female from H3. K, neck region. L, posterior genital branch and vagina. M, vaginal region. Scale bar: $10 \mu \mathrm{m}$.

() 2018 The Linnean Society of London, Zoological Journal of the Linnean Society, 2019, 185, 656-692 

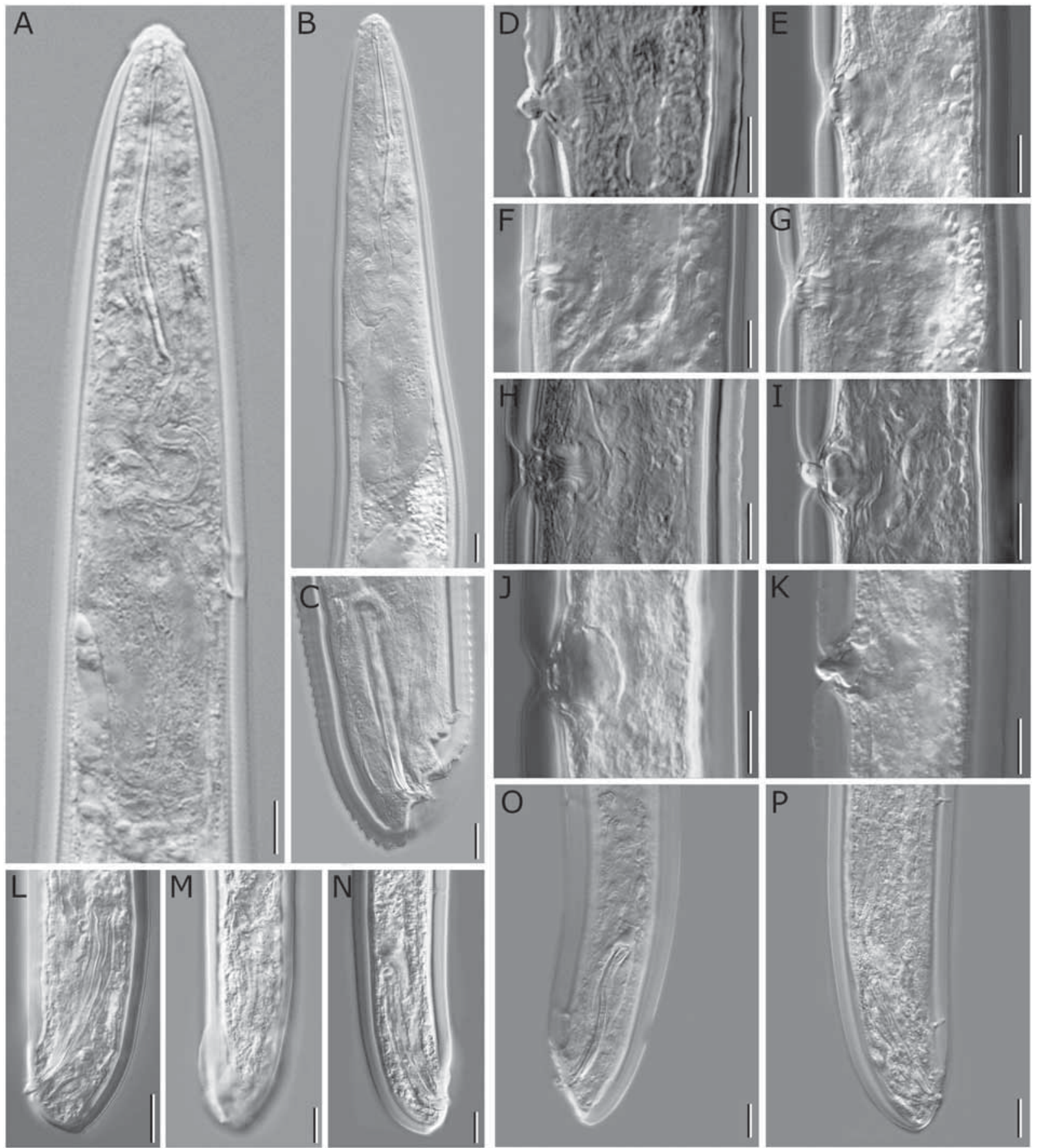

Figure 6. Light micrographs of Paratrichodorus hispanus, other localities. A-C, L-P, males from AR100. A, B, neck region. $\mathrm{C}, \mathrm{L}-\mathrm{P}$, posterior body region showing copulatory apparatus or bursa. D-K, females from AR100. D-J, vagina region. Female from H18. K, vagina with copulatory plug. Scale bars: $10 \mu \mathrm{m}$.

wild olive at Almadén de la Plata, Sevilla province, southern Spain, were deposited in the following nematode collections: IAS-CSIC (slide numbers AR110-1-AR110-4); and AR110-5 male paratypes at the USDA Nematode Collection, Beltsville, MD, USA (collection number T-7048p).
Etymology: The species epithet refers to Almadén de la Plata, the type locality where the type specimens were collected.

Description of male: Body appearance straight or slightly curved upon fixation, medium-sized body 


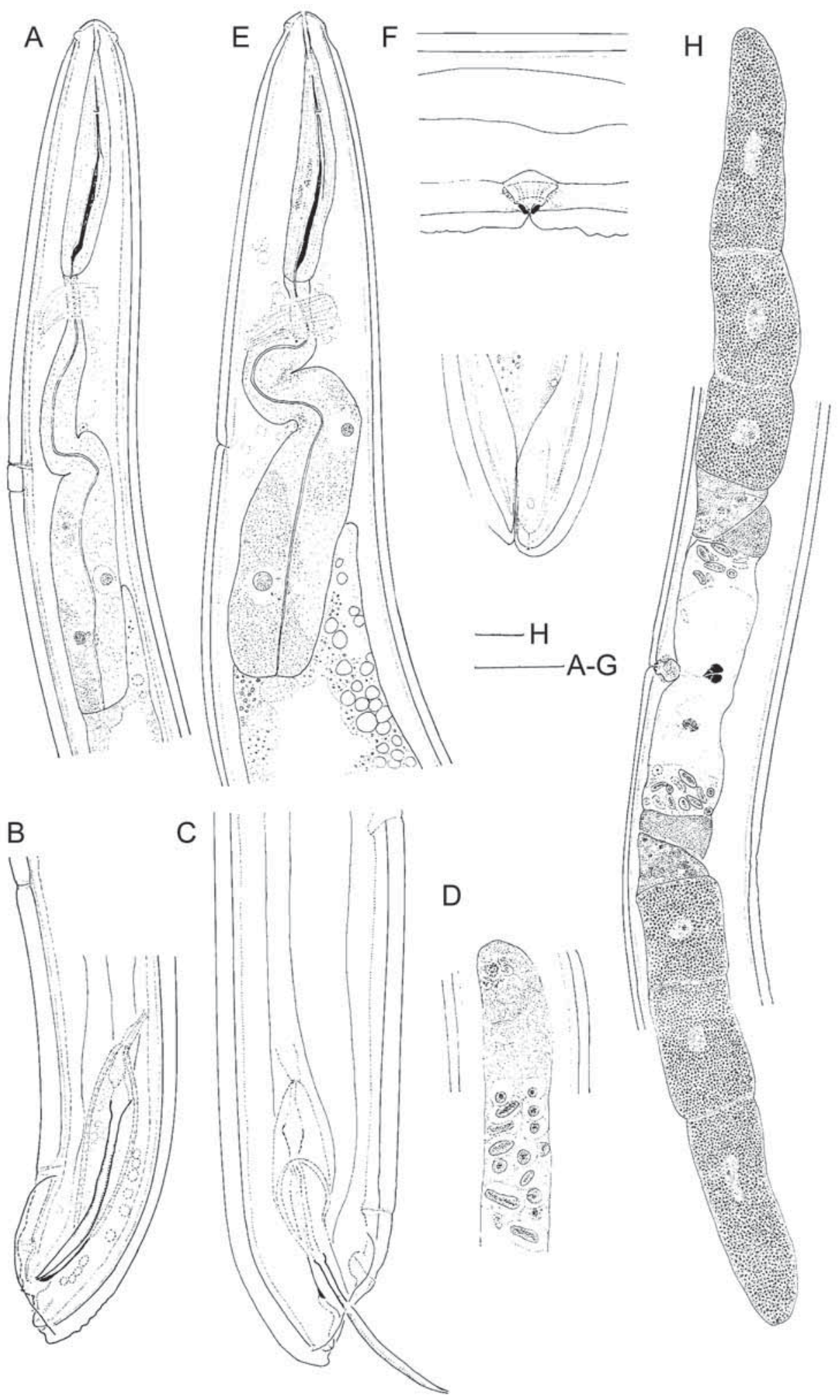

Figure 7. Line drawings of Paratrichodorus almadenensis sp. nov. A-H, male paratypes. A, neck region. B, C, posterior body region. D, anterior testis. $\mathrm{E}-\mathrm{H}$, female, holotype. E, neck region. F, vaginal region. $\mathrm{G}$, tail region. $\mathrm{H}$, female reproductive system. Scale bars: $20 \mu \mathrm{m}$ in A-G; $10 \mu \mathrm{m}$ in $\mathrm{H}$. 

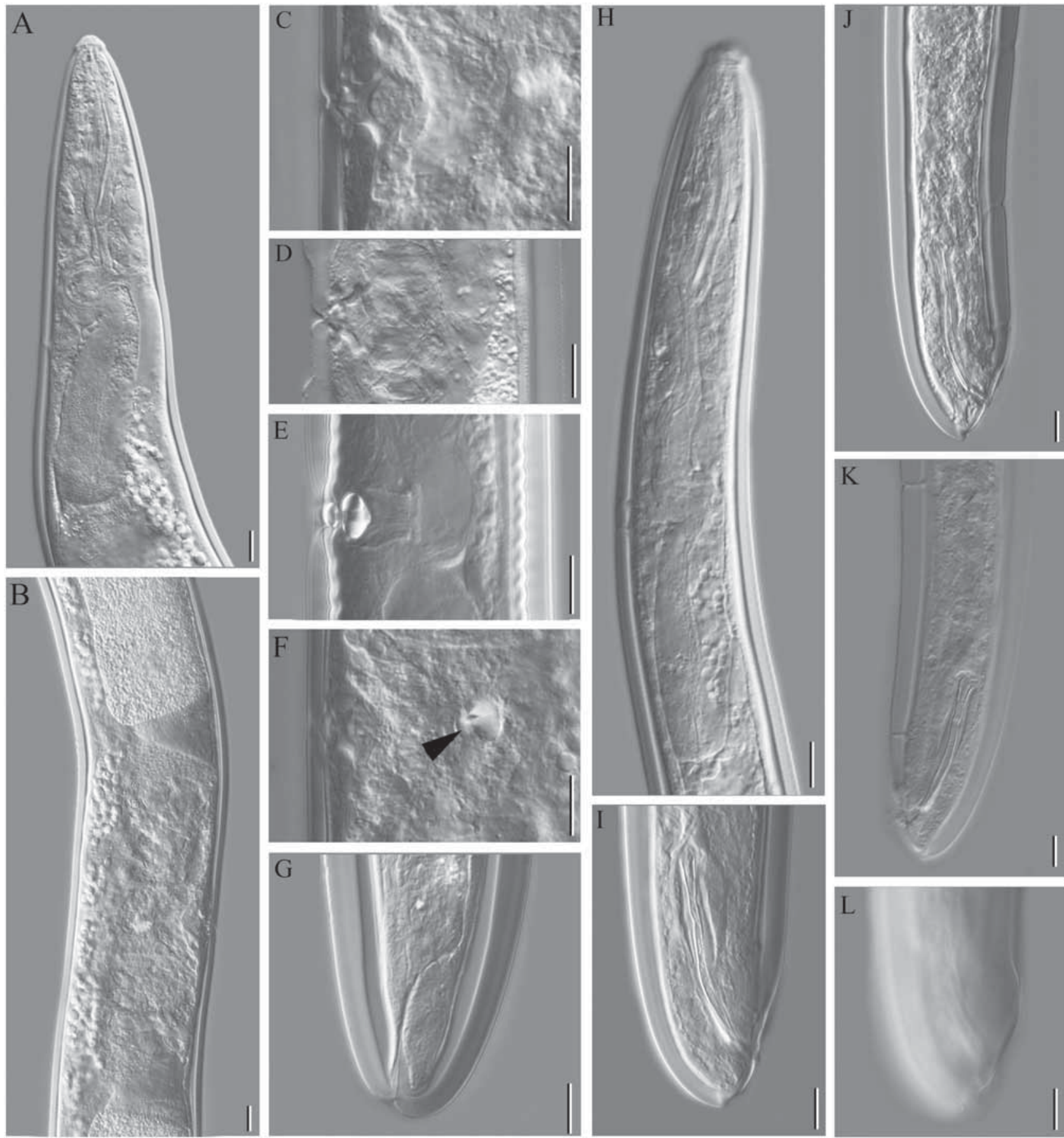

Figure 8. Light micrographs of Paratrichodorus almadenensis sp. nov. A-G, females. A, neck region, paratype. B, vaginal region, paratype. $\mathrm{C}$, vaginal region, paratype. $\mathrm{D}$, vaginal region, holotype. $\mathrm{E}$, vaginal region with copulatory plug. $\mathrm{F}$, copulatory plug at level of uterus (arrowhead). G, tail region, paratype. H-L, males, paratypes. H, neck region. J-L, posterior body region (L, bursa). Scale bars: $10 \mu \mathrm{m}$.

(average $813 \mu \mathrm{m}$ ); largely as in female. Onchiostyle medium-sized (average $47 \mu \mathrm{m}$ ) with onchium about half as long; pharynx with five gland nuclei, with the dorsal nucleus located at anterior bulb region, the two posterior ventrosublateral gland nuclei in the posterior third of the bulb, and the bulb offset or with developed dorsal intestinal overlap ( $34 \mu \mathrm{m} ; 36 \mu \mathrm{m}$ in two males); pharynx often retracted. One ventromedian cervical 
Table 5. Morphometrics of Paratrichodorus almadenensis sp. nov. from wild olive at Almadén de la Plata (Sevilla, Spain)

\begin{tabular}{|c|c|c|c|}
\hline & $\begin{array}{l}\text { Holotype } \\
\text { | female (AR110) }\end{array}$ & $\begin{array}{l}\text { Paratype } \\
\text { Females (AR110) }\end{array}$ & $\begin{array}{l}\text { Paratype } \\
\text { Males (AR110) }\end{array}$ \\
\hline$N$ & 1 & 3 & 11 \\
\hline $\mathrm{L}$ & 880 & $\begin{array}{l}740-950 \\
811.3 \pm 98.1\end{array}$ & $\begin{array}{l}735-910 \\
813 \pm 57.8\end{array}$ \\
\hline Onchium & 22.5 & $\begin{array}{l}20.0-25.5 \\
22.75 \pm 2.8\end{array}$ & $\begin{array}{l}21.0-27.5 \\
22.3 \pm 2.9\end{array}$ \\
\hline Onchiostyle & 48.0 & $\begin{array}{l}44.5-54 \\
49.3 \pm 4.8\end{array}$ & $\begin{array}{l}42.0-52.0 \\
46.7 \pm 3.4\end{array}$ \\
\hline Pharyngostom & 63.0 & $\begin{array}{l}59.0-67.0 \\
63.0 \pm 4.0\end{array}$ & $\begin{array}{l}47.5-72.0 \\
57.7 \pm 7.0\end{array}$ \\
\hline Pharynx (along) & 208.0 & $\begin{array}{l}179-187 \\
183 \pm 4.0\end{array}$ & $\begin{array}{l}125-175 \\
153.1 \pm 23.0\end{array}$ \\
\hline Anterior to guide ring & 21.5 & $\begin{array}{l}20.5,21.5 \\
(N=2)\end{array}$ & $\begin{array}{l}15.0-29.0 \\
21.5 \pm 3.3\end{array}$ \\
\hline Anterior to nerve ring & 68.0 & $\begin{array}{l}64.0 \\
(N=1)\end{array}$ & $\begin{array}{l}59.0-71.0 \\
65.3 \pm 4.9\end{array}$ \\
\hline Anterior to CP1/anterior to S-E pore in female & 131.0 & $\begin{array}{l}67.5,123.0 \\
(N=2)\end{array}$ & $\begin{array}{l}86.0-110.0 \\
96.0 \pm 7.0\end{array}$ \\
\hline Distance CP1-S-E pore & - & - & $\begin{array}{l}3.0-7.0 \\
4.7 \pm 1.2\end{array}$ \\
\hline Anterior to LP & - & - & $\begin{array}{l}54.0-101.0 \\
100.4 \pm 7.7\end{array}$ \\
\hline mbd at cardia & 49.0 & $\begin{array}{l}37.0-42.0 \\
38.7 \pm 2.4\end{array}$ & $\begin{array}{l}29.0-45.0 \\
36.5 \pm 5.62\end{array}$ \\
\hline mbd mid-body & 52.0 & $\begin{array}{l}41.0-44.5 \\
42.8 \pm 1.4\end{array}$ & $\begin{array}{l}33.0-53.0 \\
43.1 \pm 5.7\end{array}$ \\
\hline abd/o: length of anterior ovary & 200.0 & $\begin{array}{l}68.0,96.0 \\
(N=2)\end{array}$ & $\begin{array}{l}17.0-25.0 \\
20.58 \pm 2.3\end{array}$ \\
\hline Spicule length/ $\%:$ length of anterior branch & 454 & $\begin{array}{l}182,325 \\
(N=2)\end{array}$ & $\begin{array}{l}42.0-52.0 \\
48.0 \pm 2.9\end{array}$ \\
\hline Gubernaculum/ $\uparrow:$ length of posterior ovary & 200 & $\begin{array}{l}65,90 \\
(N=2)\end{array}$ & $\begin{array}{l}10-14 \\
11.7 \pm 1.1\end{array}$ \\
\hline Distance SP1-cloacal opening/\% length of posterior branch & 471 & $\begin{array}{l}248,304 \\
(N=2)\end{array}$ & $\begin{array}{l}7.5-11.0 \\
8.88 \pm 1.21\end{array}$ \\
\hline Distance SP1-SP2/o length of vagina & 6.5 & $\begin{array}{l}10.5,11.5 \\
(N=2)\end{array}$ & $\begin{array}{l}11-19 \\
15.5 \pm 2.6\end{array}$ \\
\hline Distance SP2-SP3/o vagina as a percentage of cbw & 14.0 & $\begin{array}{l}28.4 \\
(N=1)\end{array}$ & $\begin{array}{l}40-92 \\
64.4 \pm 17.0\end{array}$ \\
\hline Tail/@ G1 & 51.5 & $\begin{array}{l}24.5,34.2 \\
(N=2)\end{array}$ & $\begin{array}{l}6.5-14.5 \\
11.2 \pm 2.6\end{array}$ \\
\hline a & 17.0 & $\begin{array}{l}17.3-21.4 \\
18.9 \pm 1.8\end{array}$ & $\begin{array}{l}16.7-22.9 \\
19.1 \pm 1.9\end{array}$ \\
\hline $\mathrm{b}$ & 4.2 & $\begin{array}{l}4.0,4.2 \\
(N=2)\end{array}$ & $\begin{array}{l}4.4-6.3 \\
5.5 \pm 0.6\end{array}$ \\
\hline $\mathrm{c} / \% \mathrm{~V}$ & 53.4 & $\begin{array}{l}52.3-57.6 \\
54.8 \pm 2.2\end{array}$ & $\begin{array}{l}85.3-117.3 \\
93.7 \pm 10.8\end{array}$ \\
\hline T/@ G2 & 53.4 & $\begin{array}{l}32.0,33.4 \\
(N=2)\end{array}$ & $\begin{array}{l}60.5-98.8 \\
72.8 \pm 12.8\end{array}$ \\
\hline$c^{\prime}$ & - & - & $\begin{array}{l}0.4-0.7 \\
0.5 \pm 0.1\end{array}$ \\
\hline $\mathrm{S}-\mathrm{E}$ pore as a percentage of $\mathrm{L}$ from anterior end & 62.7 & $\begin{array}{l}9.1-16.6 \\
12.8 \pm 3.7\end{array}$ & $\begin{array}{l}11.0-14.6 \\
11.3 \pm 1.0\end{array}$ \\
\hline S-E pore as a percentage of pharynx from anterior end & 23.6 & $\begin{array}{l}37.7-65.8 \\
51.7 \pm 14.0\end{array}$ & $\begin{array}{l}49.0-86.1 \\
67.8 \pm 11.4\end{array}$ \\
\hline
\end{tabular}

Measurements are in micrometres and in the form: range and mean $\pm \mathrm{SD}$.

Abbreviations: a, body length/maximal body width; abw, anal body width; b, body length/pharyngeal length; c, body length/tail length; $c^{\prime}$, tail length/ body width at anus; cbw, cloacal body width; CP, ventromedian cervical papilla; CP1, anterior ventromedian cervical papilla; G1 and G2, (anterior and posterior gonad length, respectively/body length) $\times 100$; L, total body length; LP, labial papilla; mbw, maximal body width; $N$, number of specimens studied; S-E pore, excretory pore; SP1, SP2 and SP3, posterior, second and third precloacal supplements, respectively; T (distance from cloacal aperture to anterior end of testis/body length) $\times 100 ; \mathrm{V}$ (distance from anterior end to vulva/body length) $\times 100$. 
papilla (CP) shortly (4.5 $\mu \mathrm{m}$ average) anterior to S-E pore located opposite mid-pharyngeal bulb. Lateral cervical pores (LP) varying in position from clearly anterior to CP to shortly posterior to S-E pore. Males monorchic, with testes on average $247 \mu \mathrm{m}$ long and sausage-shaped sperm nucleus $8.5 \mu \mathrm{m} \times 2.5 \mu \mathrm{m}$ in size. Medium-sized spicules (average $48 \mu \mathrm{m}$ ) with an irregular undulated calomus (average $1.5 \mu \mathrm{m}$ wide), and blade maximal width $2.5 \mu \mathrm{m}$ (rarely $3 \mu \mathrm{m}$ ). Three precloacal supplements (SP); one exception with four SP with SP3 immediately anterior to retracted spicule, and SP1 and SP2 spread along distal half of spicules when retracted. A pair of subterminal postcloacal supplements adjacent to a pair of caudal pores. Tail half the anal body width in length.

Description of female: General appearance as in male apart from secondary sexual features. Body appearance straight or slightly curved upon fixation; mediumsized body (average $810 \mu \mathrm{m}$ ). Body cuticle swollen (3.0-3.5 $\mu \mathrm{m})$. Amphid with wide transverse aperture located immediately posterior to the outer crown of anterior sensilla; fovea stirrup-shaped, and amphidial canal often clearly visible. Onchiostyle medium-sized (average $49 \mu \mathrm{m}$ ) with onchium about half as long; stoma narrow; pharynx rather long (63 $\mu \mathrm{m}$ average), with gland nuclei (dorsal and posterior ventrosublateral pair) clearly separated and bulb offset or with clear dorsal intestinal overlap ( $36 \mu \mathrm{m}$, only in holotype). Ventromedian cervical papilla; lateral cervical pores similar to those described in male specimens. Nerve ring is adjacent to the base of the pharyngostom. Reproductive system didelphic-amphidelphic, about equally developed reflexed ovaries, finely granular oviduct cells at tip ovary; sperm stored in spermatheca adjacent to oviduct; uterus without marked ovejector; vagina $-30 \%$ of corresponding body width in length, trapezoid or more or less indented mid-way; vaginal sclerotized pieces (pars refringens vaginae) in optical section small $(1.5 \mu \mathrm{m})$, rounded triangular to oval, obliquely oriented with tips very close $(0.75 \mu \mathrm{m})$; vulva pore-like in ventral view. Well-developed sclerotized plug observed in vagina and in the uterus (Fig. 8). No lateral body pores observed. Tail minute; anus subterminal, and a pair of caudal pores present.

Diagnosis and relationships: Paratrichodorus almadenensis sp. nov. is characterized by a mediumsized body $(\sim 800 \mu \mathrm{m})$, onchiostyle (average $46.5 \mu \mathrm{m}$ in male, $49.0 \mu \mathrm{m}$ in female) and spicules (average $48.0 \mu \mathrm{m}$ ), narrow undulated calomus and rather slender blade (maximal width $2.5 \mu \mathrm{m}$ ); both posterior-most supplements (SP1 and SP2) spread along posterior half of spicule; in female, by size and shape of vaginal sclerotized pieces, small $(1.5 \mu \mathrm{m})$, rounded triangular to oval and close distal tips, vagina variable but mostly trapezoid and absence of lateral body pores except for one female with one pore anterior to vulva. Furthermore, the pharyngeal bulb can be offset in a few specimens.

The new species most closely resembles $P$. hispanus (types) but has a slightly shorter onchiostyle and spicule length in the male and no lateral body pores in the female; also, the size and position of the vaginal sclerotized pieces appear a bit different, i.e. being slightly smaller and closer together in $P$. almadenensis sp. nov. A comparison of all species within the $P$. hispanus group is presented in Table 2, separately for males and females; and specific D2-D3 expansion domains of the 28S rRNA gene, ITS-rRNA, 18S-rRNA gene and coxI sequences are deposited in GenBank with accession numbers MG739529-MG739567, MG739659-MG739670, MG739674-MG739683 and MG726826-MG726829, respectively. Morphologically and morphometrically, $P$. almadenensis sp. nov. can be distinguished from the most similar species by a number of particular characteristics resulting from its specific alphanumeric codes (in parentheses are exceptions) adapted from Decraemer \& Baujard (1998) with, in males, code L4 split up in L4 (constriction) and L5 (blade with narrower part mid-way), L6 (mid-way septum present), L7 (distal tip divided by septum) and L8 (anterior part or calomus with irregular outline): (1) for females = A222 (average, minimum, maximum), B22, C1, D1, E300, F33 (1), G1, H88 (6), I11, J11, K200, L1, M1, N1, O11, P11, Q4, R22, S1, T1; and (2) for males = A222 (average, minimum, maximum), B22, C22, D1, E0, F3 (4), G22, H33, I33, J120, K33, L88, M270, N11, O100, P1.

\section{PARATRICHODORUS RAMBLENSIS SP. NOV.}

$$
\text { (Figs 9, 10; TABLES 2, 6) }
$$

urn:lsid:zoobank.org:act:0FB545D8-3607-4B6A-845072B0CA721DA8

Holotype: Female extracted from soil samples collected from rhizosphere of grapevine at La Rambla,

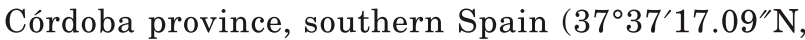
$4^{\circ} 42^{\prime} 13.03^{\prime \prime} \mathrm{W}$ ) by J. Martín Barbarroja and G. León Ropero, mounted in pure glycerine and deposited in the Nematode Collection of Ghent University, Ghent, Belgium (slide number UGMD 104343).

Paratypes: Male and female paratypes extracted from soil samples collected from the rhizosphere of grapevine at La Rambla, Córdoba province, southern Spain, were deposited in the following nematode collections: IAS-CSIC (slide numbers IASN2017_2_ M157-03-IAS_M157-05); and two male paratypes at the USDA Nematode Collection, Beltsville, MD, USA 

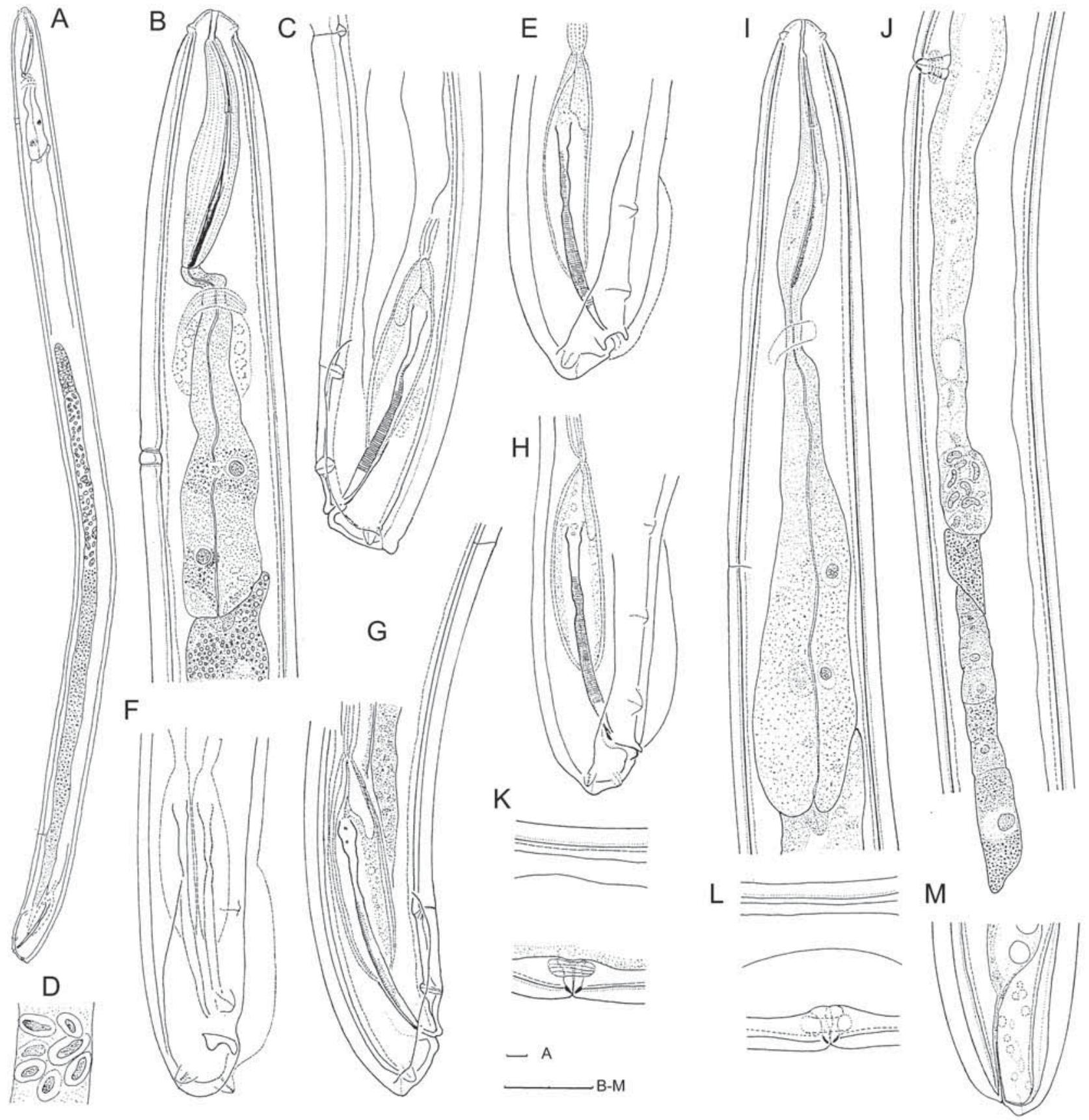

Figure 9. Line drawings of Paratrichodorus ramblensis sp. nov. A-H, males. A, total view of holotype. B, neck region of holotype. C, posterior body region of holotype. $\mathrm{D}$, sperm cells in testis, paratype male. $\mathrm{E}-\mathrm{H}$, posterior body region ( $\mathrm{E}, \mathrm{F}$ in oblique ventral view). I-M, female paratypes. I, neck region. J, vagina and posterior genital branch. K, L, vaginal region. M, tail region. Scale bars: $20 \mu \mathrm{m}$.

(collection number T-7049p). Additional populations were collected in wild olive at Antequera, Málaga province, in cultivated olive at Dos Hermanas, Sevilla province, in cultivated olive at Setenil de las Bodegas, Cádiz province, and in black alder at Andújar, Jaén province, and deposited in the Nematode Collection of IAS-CSIC.

Etymology: The species epithet refers to La Rambla, the type locality where the type specimens were collected. 

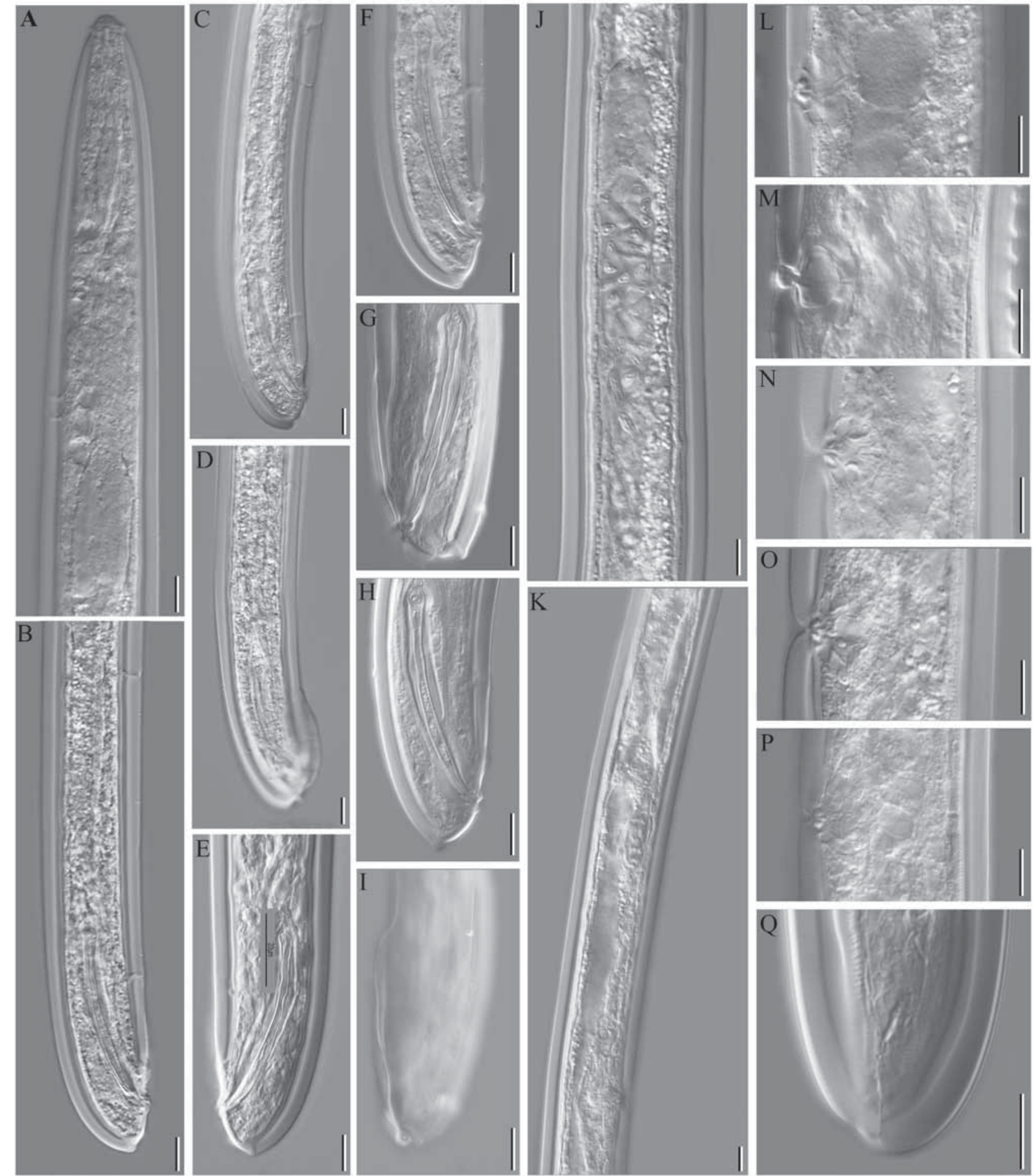

Figure 10. Light micrographs of Paratrichodorus ramblensis sp. nov. A-J, males from ST83. A, neck region. B-F, posterior body region. J, testis. K-Q, Females from ST83. K, vagina and anterior genital branch. L, O, P, vaginal region. Q, tail region. G-I, male type specimens. G, H, posterior body region, holotype and paratype respectively. I, bursa, paratype. L, M, Female, paratypes, vaginal region, without and with copulatory plug respectively. Scale bars: $10 \mu \mathrm{m}$. 
Table 6. Morphometrics of Paratrichodorus ramblensis sp. nov. females from grapevine at La Rambla (Córdoba, Spain)

\begin{tabular}{|c|c|c|c|c|c|}
\hline & $\begin{array}{l}\text { Holotype } \\
\text { Male (M157) }\end{array}$ & $\begin{array}{l}\text { Paratype } \\
\text { Males (M157) }\end{array}$ & $\begin{array}{l}\text { Paratype } \\
\text { Females (M157) }\end{array}$ & $\begin{array}{l}\text { Males } \\
\text { (ST083) }\end{array}$ & $\begin{array}{l}\text { Females } \\
\text { (ST083) }\end{array}$ \\
\hline$N$ & 1 & 7 & 6 & 15 & 8 \\
\hline $\mathrm{L}$ & 845 & $\begin{array}{l}953-1145 \\
1014 \pm 85.7\end{array}$ & $\begin{array}{l}889-1189 \\
1030.8 \pm 165.9\end{array}$ & $\begin{array}{l}855-1090 \\
933.7 \pm 62.6\end{array}$ & $\begin{array}{l}880-1120 \\
981.3 \pm 73.5\end{array}$ \\
\hline Onchium & 25.0 & $\begin{array}{l}23.0-28.0 \\
25.9 \pm 1.7\end{array}$ & $\begin{array}{l}22.0-27.5 \\
25.17 \pm 2.07\end{array}$ & $\begin{array}{l}24.5-29 \\
26.6 \pm 1.3\end{array}$ & $\begin{array}{l}23.5-29.5 \\
26.2 \pm 3.0\end{array}$ \\
\hline Onchiostyle & 53.0 & $\begin{array}{l}50.0-56.0 \\
52.6 \pm 2.2\end{array}$ & $\begin{array}{l}46.0-57.0 \\
53.0 \pm 4.2\end{array}$ & $\begin{array}{l}51-55 \\
53.4 \pm 1.6\end{array}$ & $\begin{array}{l}49-53 \\
52.2 \pm 5.1\end{array}$ \\
\hline Pharyngostom & 60.0 & $\begin{array}{l}61.0-63.0 \\
62.6 \pm 1.3\end{array}$ & $\begin{array}{l}56.0-68.0 \\
63.5 \pm 4.3\end{array}$ & $\begin{array}{l}59-72 \\
64.6 \pm 2.7\end{array}$ & $\begin{array}{l}61-79 \\
64.56 \pm 5.6\end{array}$ \\
\hline Pharynx & 142.0 & $\begin{array}{l}145-161 \\
154.1 \pm 7.0\end{array}$ & $\begin{array}{l}150-181 \\
170.0 \pm 11.5\end{array}$ & $\begin{array}{l}143-175 \\
168.6 \pm 14.1\end{array}$ & $\begin{array}{l}153-174 \\
163.44 \pm 12.42\end{array}$ \\
\hline Dorsal pharyngeal overlap & 17.0 & $\begin{array}{l}10.0-22.0 \\
16.7 \pm 4.8\end{array}$ & $\begin{array}{l}11.0-15.5 \\
13.0 \pm 2.3\end{array}$ & $\begin{array}{l}3-10 \\
7.3 \pm 2.5\end{array}$ & - \\
\hline Anterior to guide ring & 23.0 & $\begin{array}{l}18.0-23.0 \\
21.4 \pm 1.6\end{array}$ & $\begin{array}{l}23.0-26.0 \\
23.33 \pm 1.4\end{array}$ & $\begin{array}{l}22.5-27 \\
24.4 \pm 1.4\end{array}$ & $\begin{array}{l}22-24.5 \\
23.2 \pm 2.1\end{array}$ \\
\hline Anterior to nerve ring & 66.0 & $\begin{array}{l}64.0-87.0 \\
73 \pm 8.5\end{array}$ & $\begin{array}{l}59.0-84.0 \\
79.2 \pm 12.6\end{array}$ & $\begin{array}{l}68-76 \\
70.7 \pm 2.7\end{array}$ & $\begin{array}{l}67-76 \\
71.8 \pm 3.8\end{array}$ \\
\hline $\begin{array}{l}\text { Anterior to CP1/anterior to } \\
\text { S-E pore in female }\end{array}$ & 93.0 & $\begin{array}{l}94-113 \\
107.7 \pm 8.1\end{array}$ & $\begin{array}{l}115-119 \\
114.2 \pm 4.4\end{array}$ & $\begin{array}{l}97-119 \\
105.4 \pm 6.3\end{array}$ & $\begin{array}{l}99-127 \\
113.1 \pm 10.1\end{array}$ \\
\hline Distance CP1-S-E pore & 4.0 & $\begin{array}{l}5.0-7.0 \\
5.7 \pm 0.8\end{array}$ & - & $\begin{array}{l}3-8 \\
5.3 \pm 1.4\end{array}$ & - \\
\hline mbd at cardia & 33.0 & $\begin{array}{l}29.0-36.0 \\
31.1 \pm 2.3\end{array}$ & $\begin{array}{l}33.0-36.0 \\
35 \pm 1.2\end{array}$ & $\begin{array}{l}31-49 \\
35.2 \pm 4.0\end{array}$ & $\begin{array}{l}29.5-38.5 \\
35.4 \pm 7.0\end{array}$ \\
\hline mbd mid-body & 35.0 & $\begin{array}{l}32.5-47 \\
35 \pm 5.3\end{array}$ & $\begin{array}{l}36.0-41.0 \\
38.0 \pm 2.1\end{array}$ & $\begin{array}{l}32-54 \\
37.4 \pm 4.9\end{array}$ & $\begin{array}{l}34.0-41.0 \\
38.1 \pm 6.1\end{array}$ \\
\hline $\begin{array}{l}\text { abd/o: length of } \\
\text { anterior ovary }\end{array}$ & 19.0 & $\begin{array}{l}18.0-22.0 \\
20.2 \pm 1.5\end{array}$ & $\begin{array}{l}41.0-84.0 \\
66.0 \pm 16.2\end{array}$ & $\begin{array}{l}19-24 \\
21.3 \pm 1.5\end{array}$ & $\begin{array}{l}23-67 \\
46.7 \pm 38.8\end{array}$ \\
\hline $\begin{array}{l}\text { Spicule length/ o: length } \\
\text { of anterior branch }\end{array}$ & 49.0 & $\begin{array}{l}50-53 \\
50.6 \pm 1.1\end{array}$ & $\begin{array}{l}178-321 \\
247.8 \pm 50.68\end{array}$ & $\begin{array}{l}49-54 \\
51.8 \pm 1.5\end{array}$ & $\begin{array}{l}173-290 \\
248.6 \pm 36.2\end{array}$ \\
\hline $\begin{array}{l}\text { Gubernaculum/o: length } \\
\text { of posterior ovary }\end{array}$ & 13.0 & $\begin{array}{l}12.0-18.5 \\
16.5 \pm 2.3\end{array}$ & $\begin{array}{l}59.0-77.0 \\
72.20 \pm 8.9\end{array}$ & $\begin{array}{l}7.5-20.0 \\
13.1 \pm 3.7\end{array}$ & $\begin{array}{l}21-67 \\
46.2 \pm 28.3\end{array}$ \\
\hline $\begin{array}{l}\text { Distance SP1-cloacal opening/ } \\
\text { ९ length of posterior branch }\end{array}$ & 11.0 & $\begin{array}{l}7.5-11.0 \\
9.4 \pm 1.1\end{array}$ & $\begin{array}{l}188-281 \\
259.6 \pm 49.1\end{array}$ & $\begin{array}{l}9-13 \\
10.5 \pm 1.1\end{array}$ & $\begin{array}{l}198-262 \\
232.7 \pm 28.6\end{array}$ \\
\hline $\begin{array}{l}\text { Distance SP1-SP2/ } \\
\text { length of vagina }\end{array}$ & 18.0 & $\begin{array}{l}16.0-26.5 \\
20.6 \pm 4.2\end{array}$ & $\begin{array}{l}10.5-12.0 \\
10.83 \pm 0.7\end{array}$ & $\begin{array}{l}18-25 \\
22.5 \pm 2.6\end{array}$ & $\begin{array}{l}10-13 \\
11.2 \pm 9.0\end{array}$ \\
\hline $\begin{array}{l}\text { Distance } \mathrm{SP} 2-\mathrm{SP} 3 / \% \text { vagina } \\
\text { as a percentage of } \mathrm{cbw}\end{array}$ & 75.0 & $\begin{array}{l}57-108 \\
85.2 \pm 21.9\end{array}$ & $\begin{array}{l}29.0-33.0 \\
29.6 \pm 2.5\end{array}$ & $\begin{array}{l}81-103 \\
91.1 \pm 5.8\end{array}$ & $\begin{array}{l}24.4-37.2 \\
29.1 \pm 24.0\end{array}$ \\
\hline Tail/o G1 & 9.0 & $\begin{array}{l}11.0-16.5 \\
13.5 \pm 2.3\end{array}$ & $\begin{array}{l}22.0-28.2 \\
24.50 \pm 2.5\end{array}$ & $\begin{array}{l}10.5-14.5 \\
12.6 \pm 1.6\end{array}$ & $\begin{array}{l}19.6-32.8 \\
25.6 \pm 4.0\end{array}$ \\
\hline a & 24.1 & $\begin{array}{l}20.5-35.2 \\
29.51 \pm 4.9\end{array}$ & $\begin{array}{l}21.2-29.7 \\
27.0 \pm 3.4\end{array}$ & $\begin{array}{l}17.5-30.9 \\
25.3 \pm 3.1\end{array}$ & $\begin{array}{l}21.5-29.4 \\
25.9 \pm 5.4\end{array}$ \\
\hline $\mathrm{b}$ & 6.0 & $\begin{array}{l}5.9-7.1 \\
6.57 \pm 0.4\end{array}$ & $\begin{array}{l}6.1-7.1 \\
6.4 \pm 0.7\end{array}$ & $\begin{array}{l}4.4-6.7 \\
5.57 \pm 0.5\end{array}$ & $\begin{array}{l}5.2-6.6 \\
6.0 \pm 0.7\end{array}$ \\
\hline $\mathrm{c} / \mathrm{P}$ & - & $\begin{array}{l}63.5-99.9 \\
77.5 \pm 17.0\end{array}$ & $\begin{array}{l}52.1-58.4 \\
53.6 \pm 2.6\end{array}$ & - & $\begin{array}{l}50.5-56.4 \\
53.1 \pm 3.4\end{array}$ \\
\hline$c^{\prime}$ & 0.5 & $\begin{array}{l}0.5-0.8 \\
0.6 \pm 0.1\end{array}$ & - & $\begin{array}{l}0.5-0.8 \\
0.6 \pm 0.1\end{array}$ & - \\
\hline $\begin{array}{l}\text { S-E pore as } \% \text { of } \mathrm{L} \text { from } \\
\text { anterior end }\end{array}$ & 11.5 & $\begin{array}{l}9.0-13.0 \\
11.2 \pm 1.2\end{array}$ & $\begin{array}{l}9.5-15.0 \\
11.7 \pm 2.3\end{array}$ & $\begin{array}{l}10.3-13.0 \\
11.2 \pm 3.1\end{array}$ & $\begin{array}{l}10.2-13.3 \\
11.6 \pm 1.3\end{array}$ \\
\hline $\begin{array}{l}\text { S-E pore as } \% \text { of pharynx } \\
\text { from anterior end }\end{array}$ & 68.3 & $\begin{array}{l}61.9-76.4 \\
72.9 \pm 5.71\end{array}$ & $\begin{array}{l}60.8-76.7 \\
67.4 \pm 6.1\end{array}$ & $\begin{array}{l}49.5-73.4 \\
61.9 \pm 17.4\end{array}$ & $\begin{array}{l}58.9-77.9 \\
68.9 \pm 10.9\end{array}$ \\
\hline $\mathrm{T} / \%$ G2 & 63.3 & $\begin{array}{l}63.2-77.7 \\
69.14 \pm 5.6\end{array}$ & $\begin{array}{l}21.4-31.4 \\
25.8 \pm 3.9\end{array}$ & $\begin{array}{l}147-200 \\
162.4 \pm 11.8\end{array}$ & $\begin{array}{l}22.3-26.6 \\
23.8 \pm 2.3\end{array}$ \\
\hline
\end{tabular}

Measurements are in $\mu \mathrm{m}$ and in the form: range and mean $\pm \mathrm{SD}$.

Abbreviations: a, body length/maximal body width; abw, anal body width; b, body length/pharyngeal length; c, body length/tail length; $\mathrm{c}^{\prime}$, tail length/ body width at anus; cbw, cloacal body width; CP, ventromedian cervical papilla; CP1, anterior ventromedian cervical papilla; G1 and G2, (anterior and posterior gonad length, respectively/body length) $\times 100$; L, total body length; LP, labial papilla; mbw, maximal body width; $N$, number of specimens studied; S-E pore, excretory pore; SP1, SP2 and SP3, posterior, second and third precloacal supplements, respectively; T, (distance from cloacal aperture to anterior end of testis/body length) $\times 100 ; \mathrm{V}$, (distance from anterior end to vulva/body length) $\times 100$ 
Description of male: Body on average $~ 1000 \mu \mathrm{m}$ long, largely cylindrical, anteriorly gradually tapered to a rounded lip region with protruding sets of four double papillae (four cephalic and two subdorsal and two subventral outer labial papillae); body cuticle swollen (3.0-4.5 $\mu \mathrm{m})$ upon fixation. Amphid with wide transverse aperture located immediately posterior to the outer crown of anterior sensilla, fovea stirrup-shaped and amphidial canal often clearly visible. Stoma narrow; strengthening rods 4.0-5.0 $\mu \mathrm{m}$ long. Pharyngostom with rather long ( $59 \mu \mathrm{m}$ average) ventrally curved onchiostyle with half as long onchium, well marked from slender mid-pharynx (isthmus). The isthmus gradually widens to a glandular elongated bulb with three of the five gland nuclei clearly visible: the dorsal nucleus located at the anterior bulb region, the two posterior ventrosublateral gland nuclei in the posterior third of the bulb. Pharyngeal bulb overlapped (10$22 \mu \mathrm{m}$ ) dorsally by intestine; cardia poorly developed. A single non-protruding ventral cephalic papilla (CP) present shortly $(4-7 \mu \mathrm{m})$ anterior to the S-E pore, located opposite the anterior portion of the pharyngeal bulb, i.e. near dorsal gland nucleus; on each side, a lateral body pore (LP) at about the level of the S-E pore. Nerve ring around narrowest part or isthmus, close to posterior base of pharyngostom. Reproductive system monorchic; short germinal zone, vesicular seminalis well developed (average $208 \mu \mathrm{m}$ long) with sperm cells with sausage-shaped nucleus $(6.7-7.5 \mu \mathrm{m} \times 2.3 \mu \mathrm{m}$ in longitudinal optical section). Spicules $\sim 50 \mu \mathrm{m}$ on average, manubrium slightly widened, calomus short, narrower (1.0-1.5 $\mu \mathrm{m})$ and undulating, continuing in a wider blade (2.0-2.5 $\mu \mathrm{m}$, except $3.0 \mu \mathrm{m})$, distally tapered, undulation may be more or less pronounced; spicules with fine transverse striation except for manubrium and distal end. Gubernaculum with slightly thickened keel, usually less than one-third of the length of the spicule. Anterior anal lip bifid. Spicular capsule of suspensor muscles narrow, elongated; copulatory muscles hardly developed. Bursa medium-sized, extending anteriorly to the level of the calomus and posteriorly to the pair of postcloacal supplements, subterminally on the tail. Three precloacal supplements: SP1 close to the cloacal opening, SP2 level with posterior end of calomus of retracted spicule or immediately posterior to it and SP3 2.5 times maximal body diameter (on average) anterior to SP2 and hardly developed. One pair of caudal pores subterminally, near postcloacal supplements. Tail shorter than anal body width.

Description of female: General appearance as in male apart from secondary sexual features. Reproductive system didelphic-amphidelphic, about equally developed reflexed ovaries, finely granular oviduct cells at tip of ovary; sperm stored in spermatheca adjacent to oviduct; uterus with hardly marked ovejector; vagina $\sim 30 \%$ of corresponding body width long, more or less indented mid-way; vaginal sclerotized pieces (pars refringens vaginae) in optical section mostly fine, drop-like, rounded triangular to oval with average size $1.9 \mu \mathrm{m}$, obliquely oriented with tips very close (average $1.2 \mu \mathrm{m}$ ); vulva pore-like in ventral view. Well-developed sclerotized plug observed in vagina (Fig. 10). No lateral body pores observed. Tail minute, anus subterminal, with a pair of caudal pores present.

Remarks: In the population of Dos Hermanas, Sevilla (ST083), one postvulvar body pore was observed in three females at 148,164 and $198 \mu \mathrm{m}$ posterior to the vulva; vaginal sclerotized pieces varied in shape and size from rounded triangular to rounded rectangular $(1.5-2.3 \mu \mathrm{m}$, average $1.7 \mu \mathrm{m})$, with tips on average $0.7 \mu \mathrm{m}$ apart (Fig. 10).

Diagnosis and relationships: Paratrichodorus ramblensis sp. nov. is characterized by a rather long and slender body [body length $(\mathrm{L})=$ average $1015 \mu \mathrm{m}$; $\mathrm{a}=29.5$ average $]$ and onchiostyle (average $52.5 \mu \mathrm{m}$ ), a pharynx with gland nuclei (dorsal and posterior ventrosublateral pair) clearly separated and with developed dorsal intestinal overlap. Males possess $50.5 \mu \mathrm{m}$ long and rather slender spicules with undulating calomus, one ventromedian cervical papilla shortly anterior to the S-E pore opposite the anterior one-third of the pharyngeal bulb, sperm cells with a large sausageshaped nucleus, and three precloacal supplements, of which two are within the region of retracted spicules; a pair of subterminal postcloacal supplements adjacent to a terminal pair of caudal pores; and a tail shorter than anal body width. Females are characterized by sperm stored in a spermatheca near the oviduct, a short vagina (about one-third of corresponding body width), mid-way indented and with fine drop-like, rounded triangular to oval $(1.9 \mu \mathrm{m})$, obliquely oriented vaginal sclerotized pieces with tips close to one another; and no lateral body pores in the type population.

The new species most closely resembles $P$. diver gens in the slender spicule shape compared with the somewhat broader spicules in $P$. almadenensis sp. nov., $P$. hispanus and $P$. anemones. It differs from $P$. divergens and from $P$. anemones by the location of the two posterior precloacal supplements, more spread in the region of the retracted spicules, i.e. similar to $P$. hispanus and $P$. almadenensis, instead of SP1 and SP2 close together. In females, the new species differs from $P$. divergens in the orientation of the vaginal sclerotized pieces with tips not diverged, and from $P$. anemones in the shape and size of the vaginal sclerotized pieces, more developed, wider triangular compared with $P$. anemones, with very fine triangular pieces in optical section and in general with a less indented vagina than in $P$. anemones. Lateral body pores appear to be 
absent in the new species (except in a non-type population) compared with usually two (one prevulvar, one postvulvar) in $P$. anemones. The new species also differs from the other $P$. hispanus-like species by the longer (average $>1000 \mu \mathrm{m}$ ) and more slender body (a $\sim 30 \mu \mathrm{m}$ on average in male), the S-E pore located a bit more posterior in the neck region (average at $73 \%$ of pharynx from anterior end in male) and the slightly larger but fine vaginal sclerotized pieces.

Specific alphanumeric codes (in parentheses are exceptions) of the polytomous key adapted from Decraemer \& Baujard (1998) are as follows: (1) for males = A323 (average, minimum, maximum), B22, C22, D11, E0, F3, G22, H33, I33, J100, K33, L88, M270, $\mathrm{N} 11, \mathrm{O} 100, \mathrm{P} 1$; and $(2)$ for females = A323, B22, C1, D1, E300, F300, G1, H66, I11, J11, K230, L12, M1, N1, O11, P11, Q4, R22, S1, T1. Variation in non-type population females in A223 and H86; males in A223.

Diagnosis: Cuticle usually not swollen when fixed. One pair of caudal pores. Female reproductive system monodelphic-prodelphic; spermatheca present; postvulvar uterine sac present, minute to large. Vagina well developed, length about half to more than one-half body width and anteriorly directed. Vaginal sclerotization small to medium-sized, often weakly sclerotized. One pair of lateral advulvar body pores present. Males with one ventromedian cervical papilla and one pair of lateral cervical pores. Spicules long, slender; without a spicule capitular extension, shaft striated, with or without bristles. Caudal alae absent or at most rudimentary, as in Monotrichodorus sacchari Baujard \& Germani, 1985. Oblique copulatory muscles moderately developed, extending to slightly anterior to the retracted spicules. Three medioventral precloacal supplements present. One pair of large subventral postcloacal papillae. Type species: Monotrichodorus monohystera (Allen, 1957) Andrássy, 1976 (syn. Trichodorus monohystera Allen, 1957; syn. Monotrichodorus acuparvus Siddiqi, 1991; syn. Monotrichodorus parvus Siddiqi, 1991; syn. Monotrichodorus proporifer Siddiqi, 1991.

\section{MONOTRICHODORUS VANGUNDYI RODRIGUEZ-M, SHER \& SIDDIQI, 1978}

(FIG. 11; TABLE 7)

Two populations of this species were collected from the rhizosphere of a forest at Aguas Zarcas, San Carlos, Alajuela and Toro Amarillo, Valverde Vega, Costa Rica.

Description of male: Body appearance slightly curved ventrally, more abruptly curved posteriorly. Cuticle not swollen after fixation. Onchiostyle large-sized (average $65 \mu \mathrm{m}$ ) with onchium about half as long; pharynx with gland nuclei (dorsal and posterior ventrosublateral pair) clearly visible and without intestinal overlap. Secretoryexcretory pore located at about the beginning of the basal pharyngeal bulb. Paired lateral cervical pores immediately posterior to the S-E pore. Large sperm cells. Three ventromedian supplementary papillae (two supplements clearly within the region of the retracted spicule; the third supplement at a quarter of the length of the spicule head). Spicules conspicuously cephalated, slender, slightly curved ventrally; no transverse striae. Gubernaculum almost linear.

Description of female: Body slightly curved ventrally. Cuticle, pharynx and S-E pore as in male. Paired lateral cervical pores immediately posterior to the amphid openings. Gonad single, with flexure at oviduct; uterus elongated, with vagina directed anteriorly. Vulva transverse slit, vaginal cuticularization conspicuous, rod-like with tips close to one another. Paired lateral body pores within one vulvar body width anterior to vulval level. Anus subterminal.

Remarks: The morphology and morphometrics of the Costa Rican populations agree closely with those of the original description from Ecuador and Panama by Rodriguez-M et al. (1978) (Table 7), except for longer body length in females and males (692-913 and $760-941$ vs. $650-810$ and $670-830 \mu \mathrm{m}$, respectively), longer onchiostyle in females and males (60.0-68.5 and $59.0-72.5$ vs. $48-57$ and $49-56 \mu \mathrm{m}$, respectively), and spicules and gubernaculum length (55.0-60.0 and $19.5-26.5$ vs. $50-57$ and $13-16 \mu \mathrm{m}$, respectively), and no transverse striae in spicules vs. striae in fixed type specimens. Nevertheless, these differences should be regarded as intraspecific geographical variation.

Monotrichodorus vangundyi was described from soil around the roots of oil palm (Elaeis guineensis Jacq.) near Rosa Zarate (Ecuador) and from citrus, banana and native forest soils near the type locality. It was also found in Rio Corutu riverbed soil, Puerto Armuelles, and around roots of the Kapok tree [Ceiba pentandra (L.) Gaertn.], Barro Colorado, Panamá (Rodriguez-M et al., 1978). This work represents the first report of the species in Costa Rica and, together with the report of M. monohystera in banana in Costa Rica (Rodriguez-M et al., 1978), confirms the Monotrichodorus species distribution in Central America, where it seems to occur in cultivated and natural habitats.

\section{MOLECULAR CHARACTERIZATION OF THE PARATRICHODORUS HISPANUS GROUP AND MONOTRICHODORUS SPECIES}

Amplification of the D2-D3 expansion domains of the $28 \mathrm{~S}$ rRNA gene, ITS1 rRNA, partial $18 \mathrm{~S}$ rRNA 

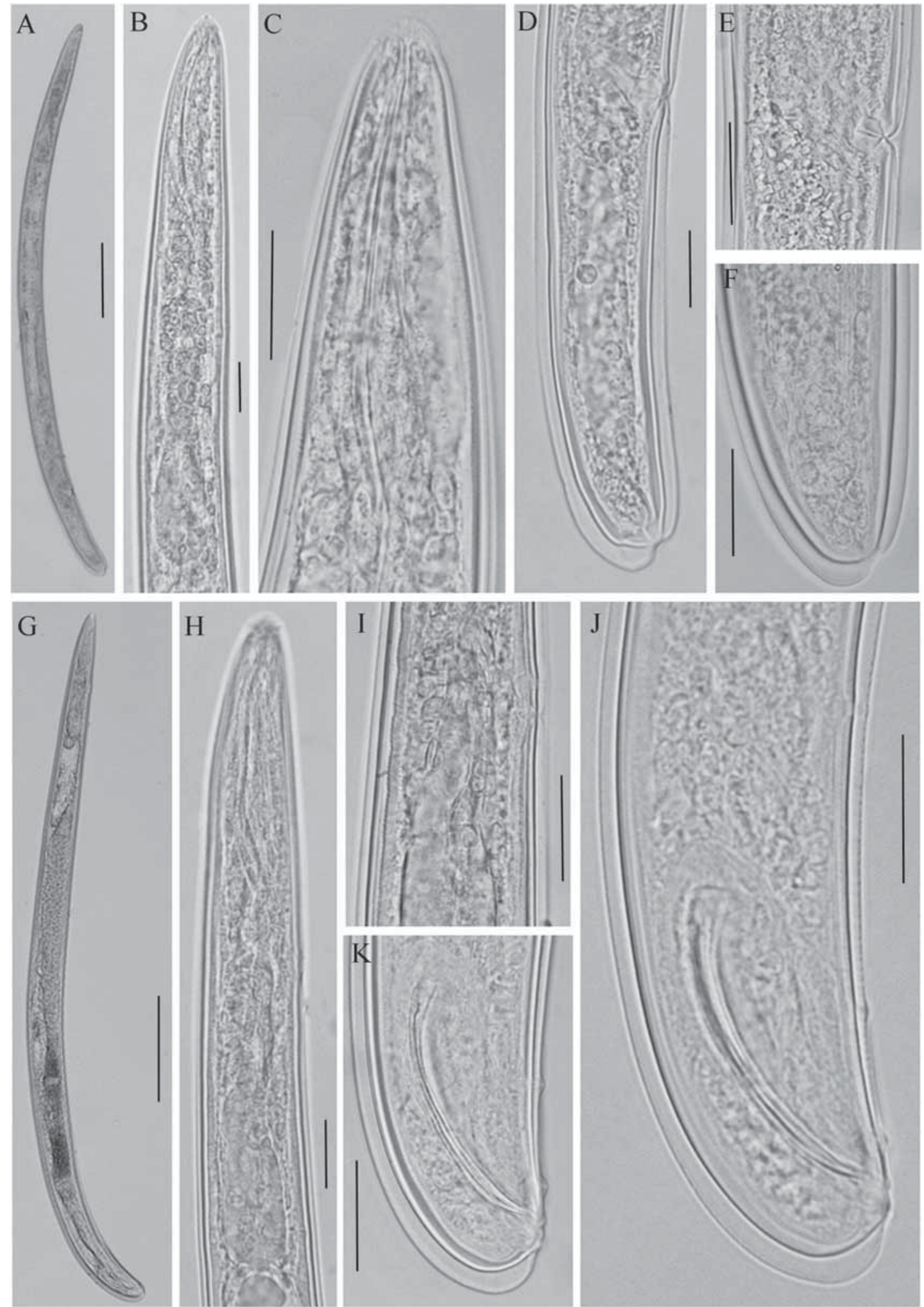

Figure 11. Light micrographs of Monotrichodorus vangundyi Rodriguez-M, Sher \& Siddiqi, 1978. A, entire female. B female neck region. $\mathrm{C}$, female lip region. D, E, vulval region. F, female tail region. G, entire male. H, I, male neck region. J, $\mathrm{K}$, male tail, Scale bars: $200 \mu \mathrm{m}$ in A, G; $20 \mu \mathrm{m}$ in B-F, H-K. 
Table 7. Morphometrics of Monotrichodorus vangundyi Rodriguez-M, Sher \& Siddiqi, 1978 females from forest at Aguas Zarcas, San Carlos, Alajuela and Toro Amarillo, Valverde Vega (Costa Rica)

\begin{tabular}{|c|c|c|c|c|}
\hline & \multicolumn{2}{|c|}{ Aguas Zarcas } & \multicolumn{2}{|c|}{ Toro Amarillo } \\
\hline & Females & Males & Female & Males \\
\hline$N$ & 4 & 9 & 1 & 2 \\
\hline $\mathrm{L}$ & $\begin{array}{l}692-800 \\
751 \pm 45\end{array}$ & $\begin{array}{l}760-905 \\
856 \pm 61.7\end{array}$ & 913 & 907,941 \\
\hline Onchiostyle & $\begin{array}{l}60.0-68.5 \\
65.4 \pm 3.9\end{array}$ & $\begin{array}{l}59.0-72.5 \\
65.2 \pm 4.8\end{array}$ & 58.0 & $59.0,67.0$ \\
\hline Onchium & $\begin{array}{l}32.5-42.0 \\
37.3 \pm 4.1\end{array}$ & $\begin{array}{l}34.5-38.5 \\
36.3 \pm 2.0\end{array}$ & 41.5 & $35.0,38.0$ \\
\hline Pharynx (along) & $\begin{array}{l}171-182 \\
176 \pm 4.9\end{array}$ & $\begin{array}{l}173-247 \\
194 \pm 24.0\end{array}$ & 200 & 202,248 \\
\hline Anterior to guide ring & $\begin{array}{l}37.5-42.5 \\
39.7 \pm 2.6\end{array}$ & $\begin{array}{l}30.5-39.0 \\
34.8 \pm 6.0\end{array}$ & 40.5 & $31.0,40.0$ \\
\hline Anterior to S-E pore & $\begin{array}{l}93.5-104.0 \\
97.5 \pm 5.7\end{array}$ & $\begin{array}{l}95.5-106.5 \\
101.3 \pm 5.5\end{array}$ & 102.5 & 97,107 \\
\hline mbd at cardia & $\begin{array}{l}29.0-45.0 \\
35.6 \pm 8.0\end{array}$ & $\begin{array}{l}28.3-36.0 \\
33.1 \pm 4.2\end{array}$ & 42.5 & $29.0,37.0$ \\
\hline mbd mid-body/vulva & $\begin{array}{l}30.0-45.0 \\
38.5 \pm 7.5\end{array}$ & - & 45.0 & $38.0,41.0$ \\
\hline Length of vagina & $\begin{array}{l}16.5-22.5 \\
20.3 \pm 2.6\end{array}$ & - & 20.5 & - \\
\hline Vagina as a percentage of cbw & $\begin{array}{l}50.0-70.7 \\
58.0 \pm 8.9\end{array}$ & - & - & - \\
\hline a & $\begin{array}{l}18.3-22.8 \\
20.8 \pm 1.9\end{array}$ & $\begin{array}{l}21.3-26.1 \\
23.5 \pm 1.5\end{array}$ & 26.5 & $22.8,23.8$ \\
\hline $\mathrm{b}$ & $\begin{array}{l}3.8-4.6 \\
4.3 \pm 0.3\end{array}$ & $\begin{array}{l}3.8-5.1 \\
4.5 \pm 0.4\end{array}$ & 4.6 & $3.8,4.5$ \\
\hline$c^{\prime}$ & $0.5(N=1)$ & $\begin{array}{l}0.4-0.6 \\
0.5 \pm 0.1\end{array}$ & 0.5 & $0.4,0.6$ \\
\hline $\mathrm{V}$ & $\begin{array}{l}84.2-87.0 \\
85.2 \pm 1.1\end{array}$ & - & 85.5 & - \\
\hline Spicule length & - & $\begin{array}{l}55.0-60.0 \\
57.6 \pm 2.2\end{array}$ & - & $58.0,60.0$ \\
\hline Gubernaculum & - & $\begin{array}{l}19.5-26.5 \\
23.0 \pm 2.4\end{array}$ & - & $23.5,26.0$ \\
\hline $\begin{array}{l}\text { S-E pore as a percentage } \\
\text { of } L \text { from anterior end }\end{array}$ & $\begin{array}{l}10.3-13.1 \\
12.0 \pm 1.5\end{array}$ & $\begin{array}{l}11.0-12.2 \\
11.7 \pm 0.6\end{array}$ & 12.3 & $10.7,11.4$ \\
\hline Anterior to CP1 & - & $\begin{array}{l}89.5-96.5 \\
93.5 \pm 3.6\end{array}$ & - & $90.0,98.0$ \\
\hline Distance CP1-S-E pore & - & $\begin{array}{l}6.0-10.0 \\
7.8 \pm 2.0\end{array}$ & - & $6.5,11.0$ \\
\hline Distance SP1-cloacal opening & - & $\begin{array}{l}7.5-10.5 \\
8.8 \pm 1.5\end{array}$ & - & $8.0,11.0$ \\
\hline Distance SP1-SP2 & - & $\begin{array}{l}19.5-22.0 \\
20.8 \pm 1.3\end{array}$ & - & $20.0,22.0$ \\
\hline Distance SP2-SP3 & - & $\begin{array}{l}41.0-47.5 \\
43.3 \pm 3.6\end{array}$ & - & $42.0,46.5$ \\
\hline
\end{tabular}

Measurements are in micrometres and in the form: range and mean $\pm \mathrm{SD}$.

Abbreviations: a, body length/maximal body width; abw, anal body width; b, body length/pharyngeal length; c, body length/tail length; $\mathrm{c}^{\prime}$, tail length/ body width at anus; cbw, cloacal body width; CP, ventromedian cervical papilla; CP1, anterior ventromedian cervical papilla; G1 and G2, (anterior, posterior gonad length, respectively/body length) $\times 100 ; \mathrm{L}$, total body length; LP, labial papilla; mbd, maximal body diameter; $N$, number of specimens studied; S-E pore, excretory pore; SP1, SP2 and SP3, posterior, second and third precloacal supplements, respectively; V, (distance from anterior end to vulva/body length) $\times 100$ 
gene and coxI regions from the new and previously known Trichodoridae spp. yielded single fragments of approximately 900, 1000, 1800 and $300 \mathrm{bp}$, respectively, based on gel electrophoresis. The D2-D3 expansion domains of the 28S rRNA gene ITS1 and coxI sequences were obtained for the first time in the present study. No molecular data were available in GenBank for the genus Monotrichodorus. The D2-D3 expansion domains of the $28 \mathrm{~S}$ rRNA gene sequences of P. almadenensis sp. nov. (MG739529-MG739530) and $P$. ramblensis sp. nov. (MG739531-MG739536) were 97\% similar (17 nucleotides and no indels) to each other and 97\% similar to P. anemones (AJ781505), with 23 nucleotides and no indels, and 21 nucleotides and no indels, respectively. The D2-D3 expansion domains of the 28S rRNA gene sequences from $P$. his panus (MG739537-MG739553) showed 91\% similarity with $P$. almadenensis sp. nov. (MG739529-MG739530) and $P$. ramblensis sp. nov. (MG739531-MG739536) and $89 \%$ similarity with several accessions deposited in GenBank, such as P. pachydermus (AM180727), with a difference of 78 nucleotides and five indels, and from $P$. anemomes (AJ781505), with a difference of 74 nucleotides and seven indels. Intraspecific variation in the D2-D3 expansion domains of the 28S rRNA gene detected in these $P$. hispanus-group species was low; zero, and from one to four nucleotides among the five populations of $P$. ramblensis sp. nov., one nucleotide between the two studied populations of $P$. almadenensis sp. nov., zero, and from one to 14 nucleotides among the 15 studied populations of $P$. hispanus. Likewise, pairwise sequence comparisons among the three Monotrichodorus species included in this study showed 93\% similarity (54 nucleotides and six indels difference) between Monotrichodorus sp. 1 (MG739558-MG739561) and $M$. vangundyi (MG739554-MG739557), 92\% similarity (63 nucleotides and two indels difference) between Monotrichodorus sp. 1 (MG739558-MG739561) and Monotrichodorus sp. 2 (MG739562-MG739567) and, finally, $90 \%$ similarity (73 nucleotides and two indels in difference) between Monotrichodorus sp. 2 (MG739562-MG739567) and M. vangundyi (MG739554-MG739557). Intraspecific variation in the D2-D3 expansion domains of the 28S rRNA gene detected in these three studied Monotrichodorus species was zero, and from one to six nucleotides among different individuals of two populations of Monotrichodorus sp. 2, and zero or four nucleotides for M. vangundyi; and zero or one nucleotide in Monotrichodorus sp. 1 populations from Ecuador.

Nine ITS1 sequences from $P$. hispanus (MG739660 MG739668) and for P. almadenensis sp. nov. were obtained in the present study, and all showed limited similarity and coverage values with the rest of the Paratrichodorus spp. deposited in GenBank. High molecular variability was detected in the ITS1 region for Paratrichodorus spp.; in fact, no similarity was found among the ITS1 sequence from $P$. almadenensis sp. nov. (MG739659) and the other ITS1 sequences of Paratrichodorus spp. deposited in GenBank using a blastn approach. Only P. macrostylus (AY430187) showed a coverage value $>50 \%$ ( $85 \%$ similarity). Molecular intra-variability was from one to 19 nucleotides for the 15 studied populations of $P$. hispanus. It was not possible to obtain any ITS1 sequences from $P$. ramblensis sp. nov., probably owing to primer-binding failure because of the variability in the primer sites. The two ITS1 sequences from $M$. vangundyi (MG739669-MG739670) did not show any similarity with accessions deposited in GenBank. Intraspecific variation of six nucleotides was found between two studied individuals.

As with the D2-D3 expansion domains of the 28S rRNA gene, $18 \mathrm{~S}$ rRNA gene sequences from $P$. almadenensis sp. nov. (MG739674-MG739675) were 99\% similar to those of $P$. ramblensis sp. nov. (MG739676MG739678) and 97\% similar to those of $P$. anemones (AF036600). The 18S rRNA gene sequences from P. hispanus (MG739679-MG739682) were 99\% similar to one accession of this species deposited in GenBank (DQ345527). Finally, one 18S rRNA gene sequence from $M$. vangundyi (MG739683) was obtained in the present study that was related, $96 \%$ similar to several Trichodorus spp., such as T. sparsus (JN123373), T. viruliferus (JN123374), T. pakistanensis (JN123369) and Trichodorus sp. CA103 (JN123375).

Four new coxI sequences from Trichodoridae spp. were sequenced in the present study. High interspecific variation was detected between the different studied species, including T. obtusus (KP984658-KP984696), and no similarity values $>60 \%$ were found among $P$. almadenensis sp. nov., $P$. ramblensis sp. nov. and $P$. hispanus. Unfortunately, owing to a shortage of Monotrichodorus sp. 1 and Monotrichodorus sp. 2 material, only the D2-D3 expansion domains of the 28S rRNA gene were sequenced; it was not possible to obtain the other studied regions (ITS1, 18S rRNA gene and $\operatorname{cox} I$ ).

\section{PHYLOGENETIC RELATIONSHIPS AMONG THE PARATRICHODORUS HISPANUS GROUP AND MONOTRICHODORUS SPECIES}

The phylogenetic relationships among the species in the family Trichodoridae inferred from analyses of the D2-D3 expansion domains of $28 \mathrm{~S}$ rRNA and the partial 18S rRNA gene sequences using BI and ML are given in Figures 12 and 13, respectively. No significant differences in topology were found using the BI or ML approaches, and only a few species in some minor clades with low bootstrap support were not 


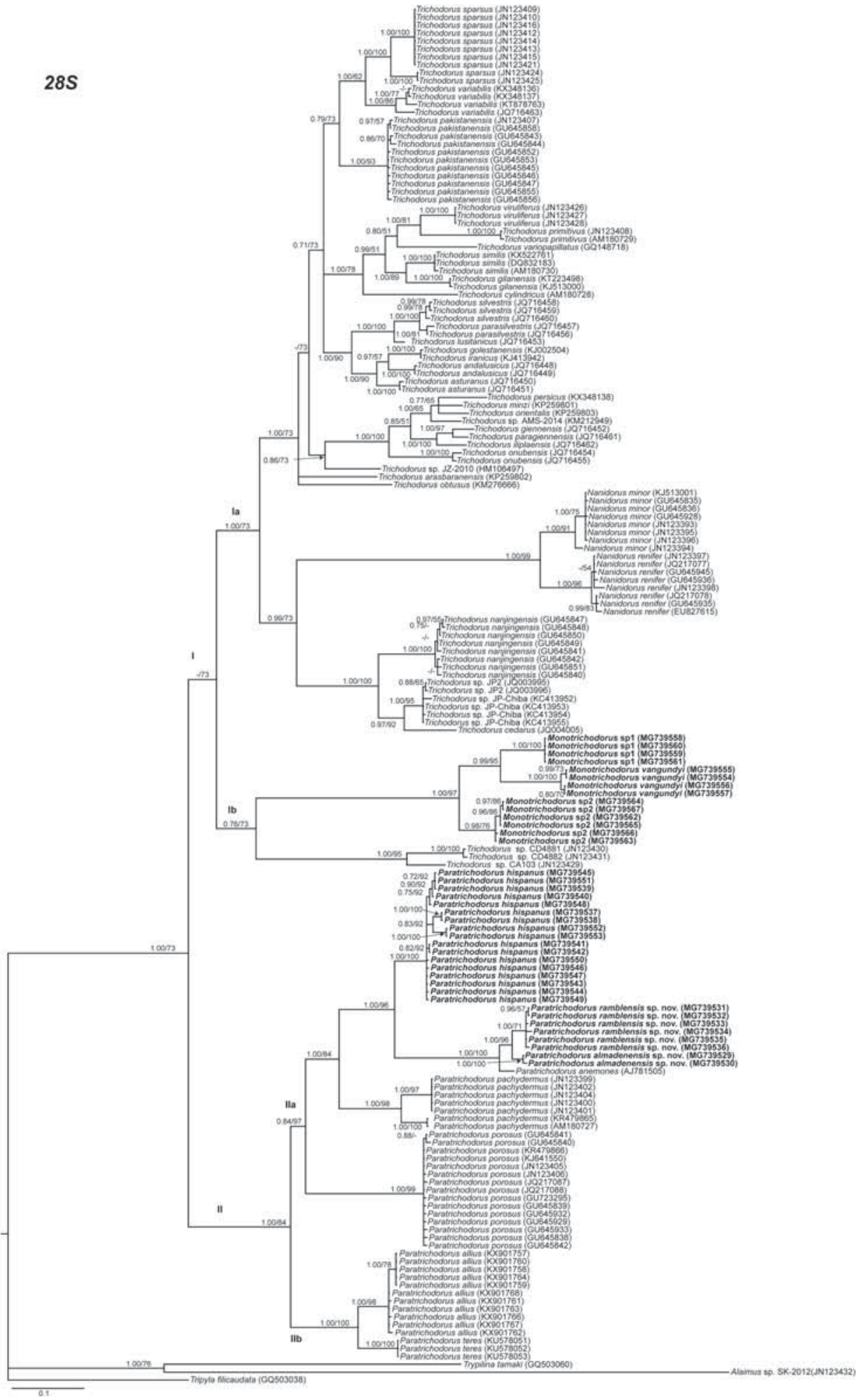

Figure 12. Phylogenetic relationships within Paratrichodorus hispanus-group complex. Bayesian 50\% majority rule consensus tree as inferred from D2-D3 expansion domains of 28S rRNA gene sequence alignment under the general time reversible of invariable sites and $\gamma$-shaped distribution $(\mathrm{GTR}+\mathrm{I}+\mathrm{G})$ model. Posterior probabilities $>0.70$ are given for appropriate clades; bootstrap values $>50 \%$ are given on appropriate clades for the maximum likelihood analysis. Newly obtained sequences in this study are in bold letters. I-II refer to the clades discussed in the main text.

(ㄷ) 2018 The Linnean Society of London, Zoological Journal of the Linnean Society, 2019, 185, 656-692 


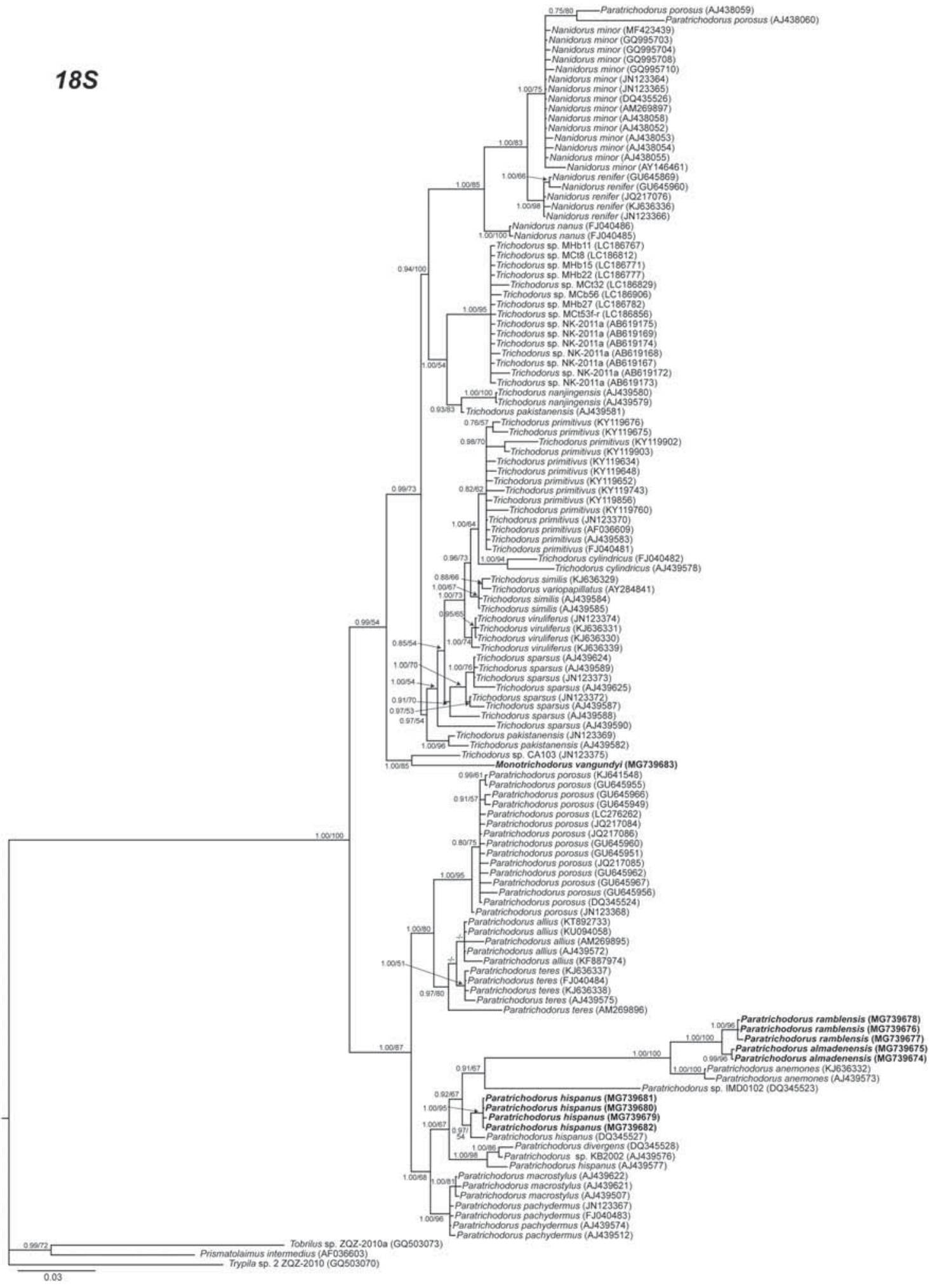

Figure 13. Phylogenetic relationships within Paratrichodorus hispanus-group complex. Bayesian 50\% majority rule consensus tree as inferred from 18S rRNA gene sequence alignment under the TIM+I+G model. Posterior probabilities $>0.70$ are given for appropriate clades; bootstrap values $>50 \%$ are given on appropriate clades for the maximum likelihood analysis. Newly obtained sequences in this study are in bold letters.

(ㄷ) 2018 The Linnean Society of London, Zoological Journal of the Linnean Society, 2019, 185, 656-692 
congruent with the general topology tree. The 50\% majority rule consensus BI and ML tree (Fig. 12) of a multiple alignment included 174 D2-D3 expansion domains of $28 \mathrm{~S}$ rRNA gene sequences and $743 \mathrm{bp}$, and revealed two major well-supported clades $(\mathrm{PP}=1.00$; BS =73), (I) formed by Trichodorus, Nanidorus and Monotrichodorus species and (II) formed by accessions from the genus Paratrichodorus including a subclade (IIa; $\mathrm{PP}=0.84 ; \mathrm{BS}=97$ ) with the two new species, $P$. almadenensis sp. nov. (MG739529-MG739530) and $P$. ramblensis sp. nov. (MG739531-MG739536), in addition to $P$. hispanus (MG739537-MG739553), $P$. anemones (AJ781505) and sequences of $P$. pachydermus and P. porosus (Fig. 12). The newly described species were clearly separated from each other and P. anemones (AJ781505). Monotrichodorus spp. combined with other unidentified Trichodorus species (JN123429-JN123431) from California formed subclade Ib, which showed a sister relationship inside the Trichodorus major clade, but its position was not well resolved $(\mathrm{PP}<0.75 ; \mathrm{BS}=73)$. The clade including all Monotrichodorus species ( $\mathrm{PP}=1.00 ; \mathrm{BS}=97$ ) formed two well-supported subclades, one of them clustering Monotrichodorus sp. 1 (MG739558-MG739561) and M. vangundyi (MG739554-MG739557) and the other with Monotrichodorus sp. 2 (MG739562-MG739567).

For the partial 18S rRNA gene, 129 sequences and $1603 \mathrm{bp}$ were included in the analyses, and this tree (Fig. 13) showed a similar topology to that of D2-D3 expansion domains of the 28S rRNA gene. All species from the $P$. hispanus group, including the accessions from P. hispanus (DQ345527 and AJ439577), clustered in the same well-supported subclade ( $\mathrm{PP}=1.00$; $\mathrm{BS}=67$ ) together with $P$. anemones (KJ636332 and AJ439573), P. divergens (DQ345528), two unidentified species (DQ345523 and AJ439576) and the two new species described here ( $P$. ramblensis sp. nov. and $P$. almadenensis sp. nov.). As in the D2-D3 expansion domains of the 28S rRNA gene tree, $M$. vangundyi (MG739683) showed a sister relationship inside the Trichodorus major clade together with Trichodorus sp. CA103 from California (JN123375), but in this case, the clade was highly supported ( $\mathrm{PP}=0.99$; $\mathrm{BS}=85$ ).

The $\mathrm{SH}$ and AU tests of different tree topologies derived from the D2-D3 expansion domains of the 28S rRNA gene support the hypothesis $(P=0.158$ and $P=0.389$, respectively) that the four genera studied (Trichodorus, Nanidorus, Monotrichodorus and Paratrichodorus) are valid, because a tree topology that places each genus in a separate clade is not significantly worse than the ML tree without constraints (Table 8). However, this result was not obtained for the partial 18S rRNA gene $(P=0.002$ and $P=0.001, \mathrm{SH}$ and $\mathrm{AU}$ test, respectively), for which the constrained tree was significantly worse than the tree without constraints. When Monotrichodorus and Paratrichodorus

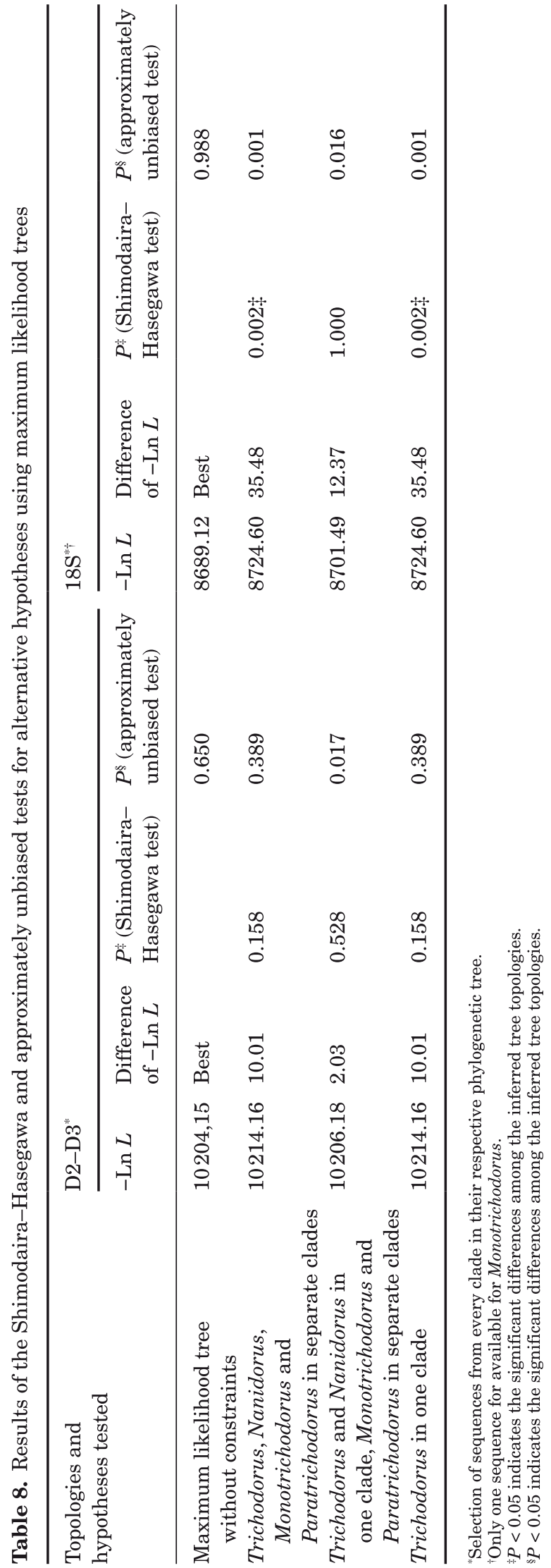


are in separate clades and Trichodorus-Nanidorus are forced to form one clade, the tree is not significantly different from the tree without constraints according to the SH test for both markers and significantly worse according to AU test. The monophyly of Trichodorus is supported in the D2-D3 expansion domains of the 28S rRNA gene marker but not for the partial 18S rRNA gene (Table 8).

\section{DISCUSSION}

The primary objective of this study was to identify and molecularly characterize species belonging to the $P$. hispanus group associated with cultivated and wild plants in southern Spain using molecular data from topotypes. Our results demonstrated that morphological studies integrated with rDNA and mitochondrial DNA molecular markers revealed the cryptic diversity of this difficult group of nematodes, particularly in the P. hispanus-group species complex. Here, we described two new species of the $P$. hispanus group based on integrative taxonomy and the phylogenetic relationships among four genera in the family Trichodoridae based on nuclear rDNA (D2D3 expansion domains of the 28S rRNA and partial $18 \mathrm{~S}$ rRNA genes). Moreover, three species of the genus Monotrichodorus were molecularly characterized in this study, which represent the first molecular data for this genus and data supporting the phylogenetic position of this monodelphic genus in relationship with Trichodorus.

\section{MORPHOLOGICAL COMPARISON OF P. HISPANUS-GROUP SPECIES COMPLEX WITH RELATED TAXA}

Together, the $P$. hispanus-group species can be distinguished easily from other Paratrichodorus species based on the shape of the spicules, which have a narrower and undulated calomus and a largely striated blade in males. In females of both new species and $P$. hispanus topotypes and in other populations, the same type of copulatory plug has been observed. These are the first observations of copulatory plugs in Paratrichodorus, and the type of mating plug clearly differs from the different types described in Trichodorus species (Decraemer, 2012). Within this species group, species differentiation based on morphological and morphometric features is subtler, because it is generally based on the observation of fixed specimens mounted on microscopic slides. As mentioned in the Introduction, Paratrichodorus species are difficult to fix, and studies illustrating the impact of fixation on morphological features are rare. Sturhan (1985) illustrated the great impact of fixation on measurements for a newly described species, Paratrichodorus weischeri Sturhan, 1985. Fixation resulted in body shrinkage of $26 \%$ of the length in males and $30 \%$ in females and was more pronounced in the posterior; the reduction of the distance between the S-E pore and the anterior end was $<19 \%$. There was also often a strong retraction of the pharynx, a shortening of the female reproductive system and of the male tail, and a reduction in the distance between the posterior-most precloacal supplements in males. The author observed only small changes in body diameter and in the lengths of the onchiostyle and spicules.

Our observations confirmed the remarks by Sturhan (1985), but we also observed a great impact on the length of the onchiostyle that was largely attributable to the less sclerotized and thus more crinkled onchiophore. The strong contraction of the pharynx also often biased the measurements of the onchiostyle because it was strongly curved, and the shape of the spicules could also be influenced by fixation. Other variations besides the impact of fixation were the variation in the number of precloacal supplements (two or four) from the regular three in a few rare specimens, which was also observed for $P$. weischeri. However, these modifications during fixation and mounting could be largely surmounted by using molecular markers, as in the present study with this group, and the use of topotypes, because all the populations clustered together phylogenetically. For these reasons, it is important to include molecular markers associated with populations for the description of new species.

\section{MOLECULAR AND PHYLOGENETIC RELATIONSHIPS IN THE PARATRICHODORUS HISPANUS GROUP}

Sequences of nuclear rRNA genes have proved to be a powerful tool for accurate molecular species identification in Trichodoridae (Boutsika et al., 2004a; Duarte et al., 2010; Decraemer et al., 2013; Ilieva-Makulec et al., 2017; Pedram et al., 2017). Our results confirm the usefulness of these markers in the $P$. hispanus group, particularly the coxI region, because these sequences provided better species delimitation for this group. However, there is no information about the coxI region from the Paratrichodorus genus; therefore, additional studies will be necessary to confirm the utility of this marker. Nucleotide differences in the coding region could provide information about the separation among species, but Shaver et al. (2016) described problems in using specific and well-designed primers for Trichodoridae species because of reaction failure and low-quality sequences. In our case, the primers that we used were different from those of Shaver et al. (2016), but some problems were found with the product amplification, and a combination of primers from different authors was used (He et al., 2005; Powers et al., 2014).

The phylogenetic relationships inferred in this study based on the D2-D3 expansion domains of the $28 \mathrm{~S}$ 
rRNA and 18S rRNA gene sequences mostly agree with the lineages obtained by Kumari \& Subbotin (2012), Pedram et al. (2017) and Ilieva-Makulec et al. (2017), except for the three species of Monotrichodorus included in the present study. The phylogenetic position of these species could also indicate that some accessions from Trichodorus spp. (JN123429-JN123431) could belong to the genus Monotrichodorus, but morphological data from these populations support the identification of this species as Trichodorus with some anatomical differences from the typical characters of the genus (S. A. Subbotin, unpublished observations). The relationship of this species with Monotrichodorus and their localities in California (Kumari \& Subbotin, 2012) and other locations in Central America (S. A. Subbotin, unpublished observations) are important clues for discovering the origin of Monotrichodorus from ancestors probably inside the genus Trichodorus. Our study is the first to provide molecular data for three species of Monotrichodorus and shows the phylogenetic relationship of Nanidorus and Monotrichodorus with the genus Trichodorus rather than with Paratrichodorus.

Our SH and AU test results showed monophyly for Trichodorus in the $28 \mathrm{~S}$ rRNA and paraphyly in the partial 18S rRNA gene. This last result is similar to that obtained by Van Megen et al. (2009) for the partial 18S rRNA gene (with a low support value) and by Kumari \& Subbotin (2012) for the D2-D3 expansion domains of the $28 \mathrm{~S}$ rRNA gene but without the $\mathrm{SH}$ or AU tests. However, is inconsistent with the results obtained by Kumari \& Subbotin (2012) for the partial $18 \mathrm{~S}$ rRNA gene. The validity of the genus Nanidorus was accepted by Duarte et al. (2010) based on morphological features and molecular analysis of the $18 \mathrm{~S}$ rRNA gene. The $\mathrm{SH}$ and AU tests validate all the tested genera (no significant difference between the tree constraining each genus to one clade and the ML tree without constraints) in the D2-D3 expansion domains of the 28S rRNA gene. However, the tests reject the validity of Nanidorus or the monophyly of Trichodorus for the partial 18S rRNA gene. The genus Monotrichodorus is accepted, but there was only one sequence for the partial $18 \mathrm{~S}$ rRNA gene, so this result should be taken with caution. These results showed the necessity of more sequences and markers to assess these questions more firmly in the future.

This work confirmed the congruence between morphological and phylogenetic data of the $P$. hispanus group, with strong support $(\mathrm{PP}=1.00 ; \mathrm{BS}=96)$ in both rRNAs (the D2-D3 expansion domains of the $28 \mathrm{~S}$ rRNA gene and the partial 18S rRNA gene). However, one sequence deposited in GenBank as P. hispanus (AJ439577) did not cluster with our sequences, so this sequence must be revised. Paratrichodorus hispanus could transmit the tobravirus Tobacco rattle virus (TRV) that causes economically important diseases, especially in potato and ornamental bulbous crops (Boutsika et al., 2004b), and the close phylogenetic association between the virus vectors $P$. anemones and $P$. divergens (Decraemer \& Robbins, 2007) and the newly described species $P$. ramblensis sp. nov. and $P$. almadenensis sp. nov. point to the possibility that these new species can be virus vectors. However, this must be tested experimentally.

\section{CONCLUSIONS}

In summary, the present study establishes the importance of using integrative taxonomic identification and highlights the time-consuming aspect and difficulty of correct identification to the species level within the $P$. hispanus group. This study also provides molecular markers for precise and unequivocal diagnosis of some species of the genus Paratrichodorus to differentiate virus-vector species, such as $P$. hispanus, with high variability.

\section{ACKNOWLEDGEMENTS}

This research was supported by grants P12-AGR 1486 and AGR-136 from 'Consejería de Economía, Innvovación y Ciencia' from Junta de Andalucía, and Union Europea, Fondo Europeo de Desarrollo regional, 'Una manera de hacer Europa', grant 219262 ArimNET ERANET FP7 2012-2015 Project PESTOLIVE 'Contribution of olive history for the management of soilborne parasites in the Mediterranean basin' from Instituto Nacional de Investigación y Tecnología Agraria y Alimentaria (INIA), grant AGL2012-37521 from 'Ministerio de Economía y Competitividad' of Spain, and grant 201740E042, 'Análisis de diversidad molecular, barcoding, y relaciones filogenéticas de nematodos fitoparásitos en cultivos mediterráneos' from the Spanish National Research Council (CSIC). A. J. Archidona-Yuste is a recipient of research contract BES-2013-063495 from 'Ministerio de Ciencia e Innovación' of Spain. C. Gutiérrez-Gutiérrez gratefully acknowledges the support of the Prometeo Project from SENESCYT, Ecuadorian Government, and from the Portuguese Foundation for Science and Technology (FCT) through a postdoctoral fellowship (SFRH/ BPD/95315/2013). The authors thank J. Martín Barbarroja and G. León Ropero from IASCSIC, Marjolein Couvreur (Ghent University), P. J. Llumiquinga (INIAP) and W. J. Enríquez (ESPE; AGROCALIDAD) for the excellent technical assistance. We would like to thank the reviewers for their thoughtful comments and efforts for improving the manuscript. 


\section{REFERENCES}

Allen MW. 1957. A review of the nematode genus Trichodorus with descriptions of ten new species. Nematologica 2: 32-62.

Almeida MTM, Almeida Santos MSN, Oliveira Abrantes IM, Decraemer W. 2005. Paratrichodorus divergens sp. n., a new potential virus vector of tobacco rattle virus and additional observations on $P$. hispanus Roca \& Arias, 1986 from Portugal (Nematoda: Trichodoridae). Nematology 7: 343-361.

Bird GW. 1967. Trichodorus acutus n. sp. (Nematoda: Diphtherophoroidea) and a discussion of allometry. Canadian Journal of Zoology 45: 1201-1204.

Boutsika K, Brown DJF, Philips MS, Blok VC. 2004a Molecular characterisation of the ribosomal DNA of Paratrichodorus macrostylus, $P$. pachydermus, Trichodorus primitivus and $T$. similis (Nematoda: Trichodoridae). Nematology 6: 641-654.

Boutsika K, Phillips MS, MacFarlane SA, Brown DJF, Holeva RC, Blok VC. 2004b. Molecular diagnostics of some trichodorid nematodes and associated Tobacco rattle virus. Plant Pathology 53: 110-116.

Colbran RC. 1956. Studies of plant soil nematodes. 1. Two new species from Queensland. Queensland Journal of Agricultural and Animal Science 13: 123-126.

Coolen WA. 1979. Methods for extraction of Meloidogyne spp. and other nematodes from roots and soil. In: Lamberti F, Taylor CE, eds. Root-knot nematodes (Meloidogyne species). Systematics, biology, and control. London: Academic Press, 317-329.

Darriba D, Taboada GL, Doallo R, Posada D. 2012. jModelTest 2: more models, new heuristics and parallel computing Nature Methods 9: 772.

Decraemer W. 1995. The family Trichodoridae: stubby root and virus vector nematodes. Dordrecht: Kluwer Academic Publishers.

Decraemer W. 2012. Tokens of love: possible diagnostic value of mating plugs and refractive secretory uterine structures in Trichodorus (Diphtherophorina: Trichodoridae). Nematology 14: $151-158$.

Decraemer W, de Almeida MTM. 1997. Identification of Trichodorus/Paratrichodorus species (Diphtherophorina). In: Santos MSNA, Abrantes IMO, Brown DJF, Lemos RM, eds. An introduction to virus nematodes and their associated viruses. Coimbra: Instituto do Ambiente e Vida, 223-272.

Decraemer W, Baujard P. 1998. A polytomous key for the identification of species of the family Trichodoridae Thorne, 1935 (Nematoda: Triplonchida). Fundamental and Applied Nematology 21: 37-62.

Decraemer W, Palomares-Rius JE, CantalapiedraNavarrete C, Landa BB, Duarte I, Almeida T, Vovlas N, Castillo P. 2013. Seven new species of Trichodorus (Diphtherophorina, Trichodoridae) from Spain, an apparent centre of speciation. Nematology 15: 57-100.

Decraemer W, Robbins RT. 2007. The who, what and where of Longidoridae and Trichodoridae. Journal of Nematology 39: 295-297.

Duarte IM, de Almeida MTM, Brown DJF, Marques I, Neilson R, Decraemer W. 2010. Phylogenetic relationships based on SSU rDNA sequences, among the didelphic genera of the family Trichodoridae from Portugal. Nematology 12: $171-180$

Hall TA. 1999. BioEdit: a user-friendly biological sequence alignment editor and analysis program for Windows 95/98/ NT. Nucleic Acids Symposium Series 41: 95-98.

He Y, Jones J, Armstrong M, Lamberti F, Moens. M. 2005. The mitochondrial genome of Xiphinema americanum sensu stricto (Nematoda: Enoplea): considerable economization in the length and structural features of encoded genes. Journal of Molecular Evolution 61: 819-833.

Holterman M, van den Wurff $A$, van den Elsen $S$, van Megen H, Bongers T, Holovachov O, Bakker J, Helder J. 2006. Phylum-wide analysis of SSU rDNA reveals deep phylogenetic relationships among nematodes and accelerated evolution toward crown clade. Molecular Biology and Evolution 23: 1792-1800.

Hooper DJ. 1962. Three new species of Trichodorus (Nematoda: Dorylaimoidea) and observations on T. minor Colbran, 1956. Nematologica 7: 273-280.

Ilieva-Makulec K, Rybarczyk-Mydłowska K, Winiszewska G, Flis L, Tereba A, Kowalewska K, Malewski T. 2017. Morphological and molecular analysis of Paratrichodorus teres (Hooper 1962) (Nematoda:Trichodoridae): a groundwork for discussion on the phylogeny and pathogenicity of Paratrichodorus species. European Journal of Plant Pathology 148: 907-917.

Jensen HJ. 1963. Trichodorus allius, a new species of stubby-root nematode from Oregon (Nemata: Dorylaimoidea). Proceedings of the Helminthological Society of Washington 30: 157-159.

Katoh K, Misawa K, Kuma K, Miyata T. 2002. MAFFT: a novel method for rapid multiple sequence alignment based on fast Fourier transform. Nucleic Acids Resources 30: 3059-3066.

Kumari S, Subbotin SA. 2012. Molecular characterization and diagnostics of stubby root and virus vector nematodes of the family Trichodoridae (Nematoda: Triplonchida) using ribosomal RNA genes. Plant Pathology 61: 1021-1031.

Loof PAA. 1965. Trichodorus anemones n. sp. with a note on T. teres Hooper, 1962 (Nematoda: Enoplida). Verslagen en Mededelingen van de Plantenziektenkundige Dienst te Wageningen 142: 132-136.

López Pérez A, Arias A, Bello A. 2001. Trichodoridae (Nematoda:Triplonchida) in Spain. Nematology 3: 403-409.

Nunn GB. 1992. Nematode molecular evolution: an investigation of evolutionary patterns among nematodes based upon DNA sequences. Unpublished $\mathrm{PhD}$. Dissertation, Nottingham: University of Nottingham.

Page RD. 1996. TreeView: an application to display phylogenetic trees on personal computers. Computer Applications in the Biosciences 12: 357-358.

Pedram M, Roshan-Bakhsh A, Pourjam E, Atighi MR, Decraemer W, Bagheri A. 2017. Additional data on Iranian trichodorids (Triplonchida: Trichodoridae) and first record of Trichodorus variabilis a rare species, Roca, 1998 Nematology 19: 121-129.

Powers TO, Bernard EC, Harris, T., Higgins R, Olson M, Lodema M, Mullin P, Sutton L, Powers KS. 2014. COI haplotype groups in Mesocriconema (Nematoda: Criconematidae) and their morphospecies associations. Zootaxa 3827: 101-146. 
Roca F, Arias M. 1986. A new Paratrichodorus species (Nematoda: Trichodoridae) from Spain. Nematologia Mediterranea 12: 181-185.

Rodriguez-M R, Sher SA, Siddiqi MR. 1978. Systematics of the monodelphic species of Trichodoridae (Nematoda: Diphterophorina) with description of a new genus and four new species. Journal of Nematology 10: 141-152.

Ronquist F, Huelsenbeck JP. 2003. MrBayes 3: Bayesian phylogenetic inference under mixed models. Bioinformatics 19: $1572-1574$.

Seinhorst JW. 1954. On Trichodorus pachydermus n. sp. (Nematoda: Enoplida). Journal of Helminthology 28: 111-114.

Seinhorst JW. 1966. Killing nematodes for taxonomic study with hot f.a. 4:1. Nematologica 12: 178.

Shaver BR, Marchant S, Martin SB, Agudelo P. 2016. 18S rRNA and COI haplotype diversity of Trichodorus obtusus from turfgrass in South Carolina. Nematology 18: 53-65.

Shimodaira H, Hasegawa M. 1999. Multiple comparisons of log-likelihoods with applications to phylogenetic inference. Molecular Biology and Evolution 16: 1114-1116.

Shimodaira H, Hasegawa M. 2001. CONSEL: for assessing the confidence of phylogenetic tree selection. Bioinformatics 17: 1246-1247.
Siddiqi MR. 1973. Systematics of the genus Trichodorus Cobb, 1913 (Nematoda: Dorylaimida), with descriptions of three new species. Nematologica 19: 259-278.

Siddiqi MR. 2002. Ecuadorus equatorius gen. n., sp. n. and Nanidorus mexicanus sp. n. (Nematoda: Trichodoridae). International Journal of Nematology 12: 197-202.

Sturhan D. 1985. Ein neuer Phytonematode aus Deutschland: Paratrichodorus weischeri spec. nov. (Nematoda, Trichodoridae). Mitteilungen aus der Biologischen Bundesanstalt für Land- und Forstwirtschoft 226: 31-45.

Subbotin SA, Waeyenberge L, Moens M. 2000. Identification of cyst forming nematodes of the genus Heterodera (Nematoda: Heteroderidae) based on the ribosomal DNA-RFLP. Nematology 2: 153-164.

Swofford DL. 2003. PAUP*: phylogenetic analysis using parsimony $\left({ }^{*}\right.$ and other methods), version $4.0 b$ 10. Sunderland: Sinauer Associates.

Van Megen H, Elsen SVD, Holterman M, Karssen G, Mooyman P, Bongers T, Holovachov O, Bakker J, Helder J. 2009. A phylogenetic tree of nematodes based on about 1200 full-length small subunit ribosomal DNA sequences. Nematology 11: 927-950.

\section{SUPPORTING INFORMATION}

Additional Supporting Information may be found in the online version of this article at the publisher's web site:

Table S1. Sequence data sets used for SH and AU tests for alternative hypotheses using ML trees for D2-D3 expansion domains of $28 \mathrm{~S}$ rRNA and 18S rRNA gene. 